\title{
Irgm1 coordinately regulates autoimmunity and host defense at select mucosal surfaces
}

Kathleen M. Azzam, ${ }^{1}$ Jennifer H. Madenspacher, ${ }^{1}$ Derek W. Cain, ${ }^{2}$ Lihua Lai, ${ }^{1}$ Kymberly M. Gowdy, ${ }^{3}$ Prashant Rai, ${ }^{1}$ Kyathanahalli Janardhan,, ${ }^{4,5}$ Natasha Clayton, ${ }^{4}$ Willie Cunningham, ${ }^{4}$ Heather Jensen, ${ }^{4}$ Preeyam S. Patel, ${ }^{6}$ John F. Kearney, ${ }^{6}$ Gregory A. Taylor, ${ }^{7}$ and Michael B. Fessler ${ }^{1}$

IImmunity, Inflammation and Disease Laboratory and ${ }^{2}$ Signal Transduction Laboratory, National Institute of Environmental Health Sciences, Research Triangle Park, North Carolina, USA. ${ }^{3}$ Department of Pharmacology and Toxicology, Brody School of Medicine, East Carolina University, Greenville, North Carolina, USA. ${ }^{4}$ Cellular \& Molecular Pathology Branch, National Toxicology Program, National Institute of Environmental Health Sciences, Research Triangle Park, North Carolina, USA. Integrated Laboratory Systems, Inc., Research Triangle Park, North Carolina, USA. ${ }^{6}$ Department of Microbiology, University of Alabama at Birmingham, Birmingham, Alabama, USA. 'Ceriatric Research, Education, and Clinical Center, Durham VA Medical Center, Durham, North Carolina, USA.

The pathogenesis of primary Sjogren's syndrome (SS), an autoimmune disease that targets the mucosa of exocrine tissues, is poorly understood. Although several mouse models have been developed that display features of SS, most of these are within the larger context of a lupus-like presentation. Immunity-related CTPase family M protein 1 (Irgm1) is an interferon-inducible cytoplasmic GTPase that is reported to regulate autophagy and mitochondrial homeostasis. Here, we report that naive $/ \mathrm{rgm}^{-1-}$ mice display lymphocytic infiltration of multiple mucosal tissues including the lung in a manner reminiscent of SS, together with IgA class-predominant autoantibodies including anti-Ro and anti-La. This phenotype persists in the germ-free state, but is abolished by deletion of $/ \mathrm{rgm} 3$. Irgm1 ${ }^{-1-}$ mice have increased local production in the lung of TECP15-idiotype IgA, a natural antibody with dual reactivity against host and pneumococcal phosphorylcholine. Associated with this, $/ \mathrm{rgm}^{1^{-1-}}$ mice display enhanced opsonization and clearance of Streptococcus pneumoniae from the lung and increased survival from pneumococcal pneumonia. Taken together, our results identify Irgm1 as a master regulator of mucosal immunity that dually modulates evolutionarily conserved self- and other-directed immune responses at the interface of host with environment.

Conflict of interest: The authors have declared that no conflict of interest exists.

Submitted: November 21, 2016 Accepted: July 11, 2017 Published: August 17, 2017

Reference information: JCI Insight. 2017;2(16):e91914. https:// doi.org/10.1172/jci.insight.91914

\section{Introduction}

Primary Sjogren's syndrome (SS) is an autoimmune disorder characterized by lymphocytic infiltration of exocrine tissues including salivary glands, lacrimal glands, and other mucosal organs such as the lungs and pancreas, in the absence of evidence of another underlying multisystem autoimmune disorder (e.g., systemic lupus erythematosus [SLE]). Among autoimmune diseases, primary SS is second in prevalence only to rheumatoid arthritis (1). Several mouse models have been developed that spontaneously display features of SS (2). While these models have advanced insight into the pathogenesis of SS, fundamental gaps persist in our understanding of the unifying mechanisms that target immune-mediated injury specifically to the epithelium of select mucosal organs.

Approximately $10 \%-20 \%$ of SS patients have clinical pulmonary involvement, but it is speculated that a much larger proportion have subclinical lung disease involving abnormal expansion of bronchus-associated lymphoid tissue (BALT) (3). BALT, a network of peribronchovascular tertiary lymphoid tissue composed of follicular DCs and organized B and T cell zones, is found in some species, but is typically absent in mice and humans except in the setting of infection, chronic lung disease, and autoimmunity (4). BALT-derived antibodies may contribute locally to progression of lung disease and also to host defense against pathogens $(5,6)$. Identification of the molecular determinants of submucosal lymphoid hyperplasia in the lung and other organs (i.e., mucosa-associated lymphoid tissue) may offer new therapeutic avenues for human disease. 
Immunity-related GTPase family M protein 1 (Irgm1) is a member of a family of interferon-inducible (IFN-inducible) cytoplasmic immunity-related GTPases (IRG) encoded by approximately 20 genes in the mouse $(7,8)$. Irgm1-null mice display dramatic susceptibility to intracellular pathogens that is thought to arise from a combination of deficient cell-autonomous resistance and infection-induced hematopoietic failure (9). Although the mechanism of action remains somewhat obscure, Irgm1 is thought to promote autophagy and mitochondrial homeostasis in part through regulating the organellar localization and activation of other IRGs (10-12). Polymorphisms in the human homolog, IRGM, have been associated with Crohn's disease (13), suggesting a possible role in restraint of spontaneous mucosal immunity against host and/or microbiota. To date, however, no role has been demonstrated for Irgm 1 in regulation of spontaneous autoimmunity.

Here, we report for the first time to our knowledge that naive $\operatorname{Irgm} 1^{-/-}$mice display lymphocytic infiltration of multiple mucosal tissues including the lung in a manner reminiscent of SS, together with IgA classpredominant autoantibodies including TEPC15-idiotype (T15-idiotype) IgA, a natural antibody with dual reactivity against host and pneumococcal phosphorylcholine (PC) (14). Associated with this, Irgm $1^{1^{--}}$mice display enhanced opsonization and clearance of Streptococcus pneumoniae from the lung. Irgm1 deletion thus reveals coordinate immune targeting of evolutionarily conserved host and pathogen epitopes at the environmental interface. Taken together, our results suggest that Irgm1 is a key regulator of mucosal immunity.

\section{Results}

Irgm $1^{-1-}$ mice have spontaneous peribronchovascular $B$ and $T$ cell infiltration. Irgm $1^{-1-}$ mice have defective host defense against several intracellular pathogens $(9,15)$. Cellular functions that have been identified as Irgm1 dependent including autophagy (13) and migration (16) also govern steady-state immune cell constitution of the lung and other organs. Given this, we examined the lungs of naive $\operatorname{Irgm1^{-1-}}$ mice and littermate controls. Remarkably, we found that 8- to 15 -week-old $\operatorname{Irgm1^{-/}}$ mice of both sexes housed under specific pathogen-free conditions had multifocal, well-formed lymphocytic aggregates in their lungs in a predominantly peribronchovascular pattern (Figure 1A). Increased lymphocytes were also found in the airway lumen of naive $\operatorname{Irgm} 1^{-1-}$ animals, as revealed by bronchoalveolar lavage (Supplemental Figure 1; supplemental material available online with this article; https://doi.org/10.1172/jci.insight.91914DS1). Immunohistochemical (IHC) staining revealed the lung parenchymal lesions to be B cell $\left(\operatorname{Pax} 5^{+}\right)$predominant, with fairly discrete $\mathrm{B}$ and $\mathrm{T}$ cell $\left(\mathrm{CD}^{+}\right.$) zones (Figure $\left.1 \mathrm{~B}\right)$, reminiscent of tertiary lymphoid tissue, i.e., BALT (4). IHC verified the presence of both $\mathrm{CD} 4^{+}$and $\mathrm{CD} 8^{+} \mathrm{T}$ cells in the lesions (Figure $1 \mathrm{C}$ ). Flow cytometry confirmed increased B cells, $\mathrm{CD}^{+} \mathrm{T}$ cells, and $\mathrm{CD}^{+} \mathrm{T}$ cells in digests of Irgm $^{-/-}$lungs (Figure $1 \mathrm{D}$ and Supplemental Figure 2), and moreover revealed that there was an increase in the $\operatorname{Irgm~}^{1^{-1}}$ lung of both conventional B2 $\left(\mathrm{CD}^{2}{ }^{+} \mathrm{CD}^{-} \mathrm{CD} 11 \mathrm{~b}^{-} \mathrm{IgM}^{10}\right)$ cells as well as B1a $\left(\mathrm{CD} 19^{+} \mathrm{CD}^{+} \mathrm{CD} 11 \mathrm{~b}^{+} \operatorname{IgM}^{\mathrm{hi}}\right)$ and $\mathrm{B} 1 \mathrm{~b}\left(\mathrm{CD} 19^{+} \mathrm{CD} 5\right.$ $\mathrm{CD}_{11 \mathrm{~b}^{+}} \mathrm{IgM}^{\mathrm{hi}}$ ) cells (Figure $1 \mathrm{E}$ and Supplemental Figure 3), innate-like B cells known to produce polyreactive natural (germline-encoded) antibodies that mediate host defense during acute infection (17).

Lymphocyte infiltration into the lung raised the possibility of a systemic lymphoproliferative disorder. However, consistent with a prior report of defective hematopoietic stem cell function in $\operatorname{Irgm1^{-/-}}$ mice (18), we found reduced numbers of lymphocytes (Figure 2, A and B, and Supplemental Figure 4A) as well as erythrocytes and platelets (Supplemental Figure 4B) in the circulation, and deficiencies in B cell (Supplemental Figure 4, C and D) and myeloid (data not shown) lineages in the bone marrow. The peritoneal cavity (PerC) is the primary anatomic site for B1 B cells, from which location these cells are thought to emigrate to the lungs and other organs in response to inflammatory insults (19). Flow cytometric characterization of cell populations in the PerC of naive $\operatorname{Irgm1^{-1-}}$ mice revealed a significant reduction in B1a cells, no change in B1b cells, and an increase in B2 cells (Figure 2C). Collectively, these results suggested dysregulation of B cell lineage development and/or trafficking in $\operatorname{Irgm} 1^{-/-}$mice.

B cells accumulate in BALT and other tertiary lymphoid organs in response to CXCL13, where local growth factors induce formation of germinal centers, sites of active B cell proliferation, differentiation, and class switching $(4,5)$. We detected increased CXCL13 in the $\operatorname{Irgm1^{-1-}}$ lung that was restricted to the cellular aggregates (Figure 3, A and B). Positive staining was also noted for B cell-activating factor (BAFF), a key B cell prosurvival and differentiation factor (1) (Figure 3, C and D). Upon more extensive profiling, we found that, although some additional cytokines were modestly increased in $\operatorname{Irgm1}^{-1-}$ lung homogenates, several cytokines that promote B cell proliferation/differentiation and/or BALT formation, including IL-2, IL-5, IL-6, IL-9, and IL-10 were not increased (Figure 3E). Nonetheless, consistent with active local cell proliferation, 

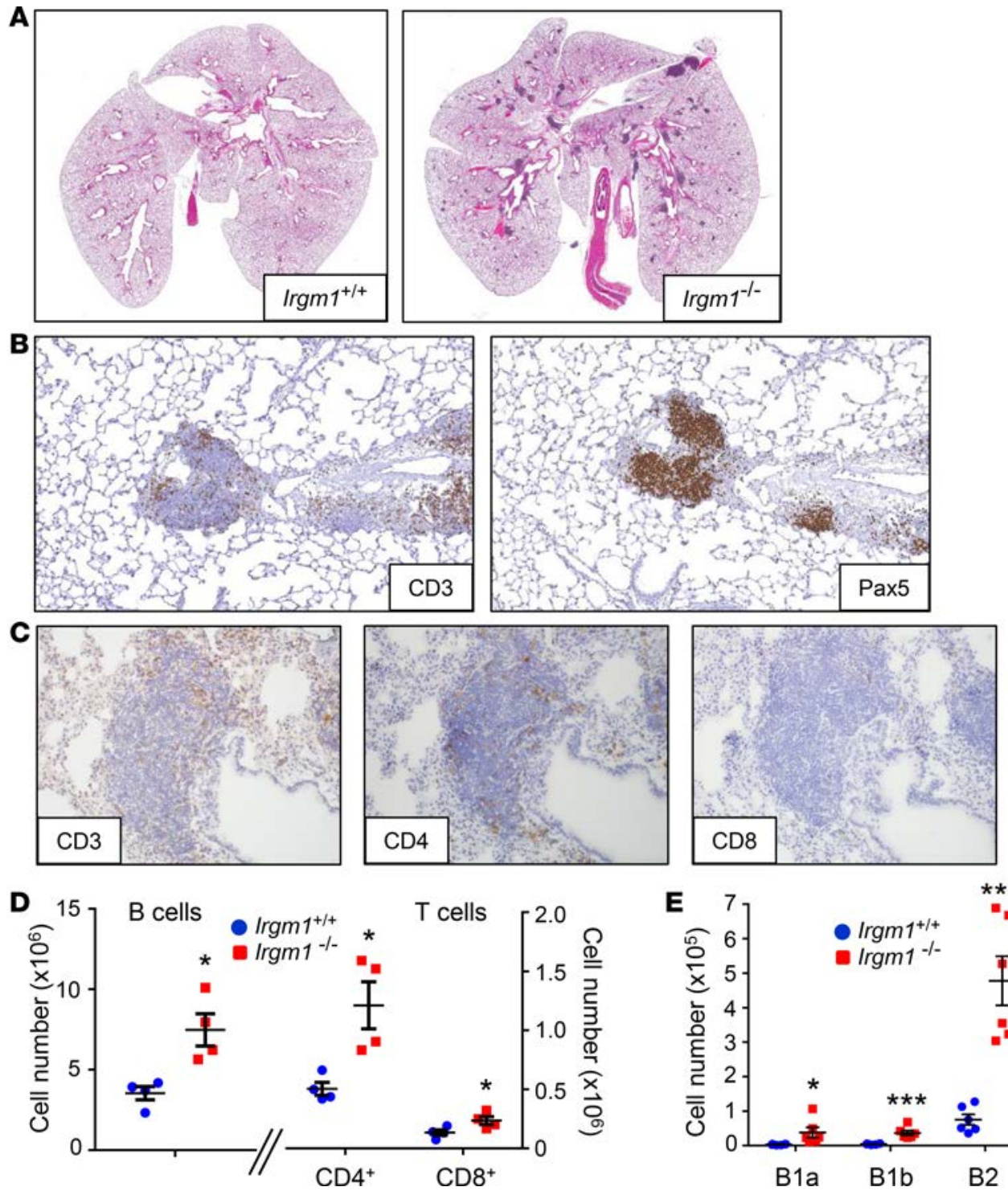

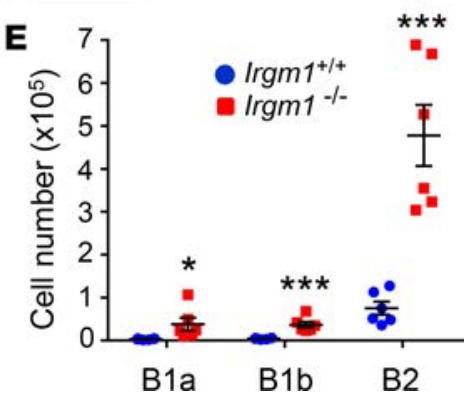

Figure 1. Peribronchovascular lymphocytic infiltrates in the Irgm $^{-1-}$ lung. (A) Hematoxylin and eosinstained lungs from naive $\mathrm{Irgm}^{-1-}$ mice and littermate controls. Original magnification, $\times 1$ 1.1. (B) Peribronchovascular cellular infiltrates in $/ \mathrm{rgm}^{-/-}$lungs were evaluated by immunohistochemical (IHC) staining for $\mathrm{CD} 3$ ( $T$ cells) and Pax5 (B cells). Original magnification, $\times 20$. (C) $/ \mathrm{rgm}^{1 /-}$ lung lesions were IHC stained for the targets shown. Original magnification, $\times 20$. (D) B cells (CD45+CD19+CD3-), and CD4+ and $\mathrm{CD8}^{+} \mathrm{T}$ cells (CD45+CD19-CD3 ${ }^{+}$) were quantified by flow cytometry in lung

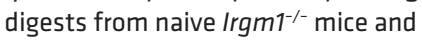
controls ( $n=4 /$ genotype). (E) B1a $\left(C^{2} 19^{+} C^{+} 5^{+} C_{11} b^{+} \operatorname{lgM}{ }^{\text {hi }}\right)$, B1b (CD19+CD5 CD11 $\mathrm{b}^{+}$IgM $\left.\mathrm{M}^{\mathrm{hi}}\right)$, and B2 (CD19+CD5-CD11 $\mathrm{b}^{-}$ $\mathrm{IgM}^{10}$ ) cells were quantified by flow cytometry in lung digests from naive Irgm $7^{-/-m i c e}$ and controls ( $n=4-6 /$ genotype). Histology and IHC data are representative of at least 4-5 mice/ genotype. Graphed data are the mean \pm SEM and are representative of at least 3 independent experiments. ${ }^{*} P<$ $0.05,{ }^{* *} P<0.001$ by unpaired 2 -tailed Student's $t$ test.

moderate numbers of lymphocytes in the lung lesions displayed positive staining for Ki-67 (Figure 3F). In addition, positive staining was noted with peanut agglutinin, a marker of activated germinal center B cells (Figure $3 \mathrm{G}$ ), and an increased frequency and number of $\mathrm{CD} 45^{+} \mathrm{B} 220^{+} \mathrm{GL} 7^{+} \mathrm{CD} 38^{-}$germinal center B cells was also noted in $\operatorname{Irgm1}^{-1-}$ lung digests by flow cytometry (Figure $3 \mathrm{H}$ and Supplemental Figure 5).

Irgm1 deletion induces lymphocytic infiltration of multiple exocrine tissues. Given the striking pulmonary phenotype, which suggested increased trafficking and tissue persistence of B cells, we extended our histopathologic screen of naive $\operatorname{Irgm}^{1^{-1-}}$ mice to more than 40 tissues (Supplemental Table 1). Lymphocytic infiltration was also noted in the salivary glands (submandibular and parotid; sublingual unaffected), extraorbital lacrimal glands, and pancreas of both sexes, with IHC confirming B and T cells in all cases (Figure 4, A-F). Associated acinar atrophy of the submandibular glands was present. In the pancreas, extensive exocrine pancreatic atrophy with adipocyte replacement and scattered infiltration of lymphocytes was present (Figure 4E). By contrast, the islets and ducts were not affected. Consistent with functional compromise of the exocrine acini of the pancreas and/or salivary glands, serum amylase was reduced in $\operatorname{Irgm1^{-/-}}$ mice (Supplemental Figure 6A). All $\operatorname{Irgm1^{-/-}}$ mice also exhibited fur loss around the eyelid margin of at least one eye (Supplemental Figure 6B), suggestive of lacrimal dysfunction.

A subset of 8- to 15 -week-old (7 of 12 males, 1 of 4 females) $\operatorname{Irgm1^{-1-}}$ mice also displayed mild chronic active inflammation in the liver. No other tissues, however, displayed any notable pathology. Examination of 9- to 14-month-old $\operatorname{Irgm1}^{-1-}$ mice of both sexes revealed marked progression of the aforementioned lung 


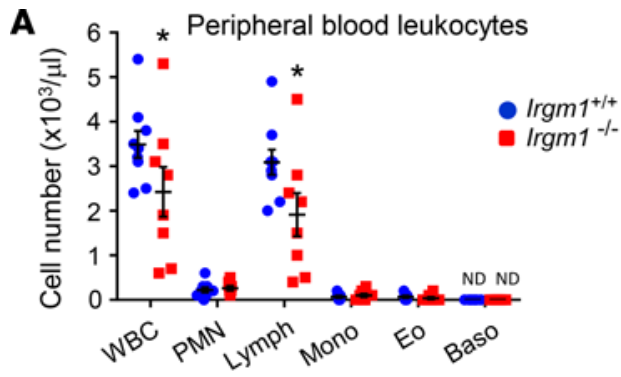

$\mathbf{B}$
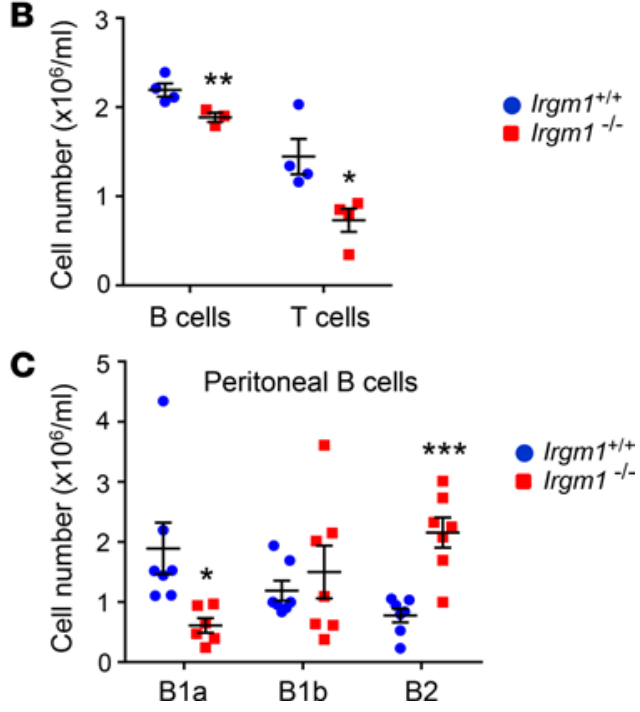

Figure 2. Dysregulated lymphocyte populations in $/ \mathrm{rgm}^{1 /-}$ mice. (A) Counts of circulating total leukocytes (WBC) and leukocyte subtypes were enumerated in naive $\mathrm{Irgm}^{-/-}$mice and controls ( $n=10$ /genotype). (B) Circulating B cells (CD3-B220+) and T cells (CD3+B220-) in naive $\mathrm{Irgm}^{-1 /-}$ mice and controls ( $n=4 /$ genotype) were quantified by flow cytometry. (C) Peritoneal B1a

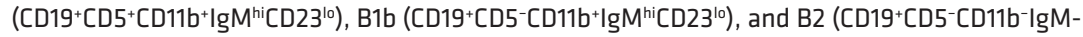
${ }^{10} \mathrm{CD} 23^{\mathrm{hi}}$ ) cells in naive $\mathrm{Irgm1^{-/- }}$ mice and controls ( $n=7 /$ genotype) were quantified by flow cytometry. Data are the mean \pm SEM and are representative of at least $2-3$ independent experiments. ${ }^{*} P<0.05,{ }^{*} P<0.01,{ }^{* * *} P<0.001$ by unpaired 2 -tailed Student's $t$ test. ND, not detected; PMN, neutrophil; Lymph, Iymphocyte; Mono, monocyte; Eo, eosinophil; Baso, basophil.

lesions with age (Supplemental Figure 7), but no involvement of additional tissues beyond those noted in younger mice. Collectively, this selective pattern of exocrine/ mucosal organ involvement in the absence of disease in organs typically involved in SLE and other autoimmune disorders (i.e., kidneys, skin, lymph nodes, central nervous system, joints) is reminiscent of primary SS in humans.

Tissue lesions of $\operatorname{Irgm1}^{-1-}$ mice persist in the germ-free state. Given that $\operatorname{Irgm1^{-1-}}$ mice have compromised immunity and BALT can be induced by infection $(4,5)$, we performed an extensive screen for bacterial, viral, and fungal infection. However, no pathogens were detected by serology (Supplemental Table 2), nor was Pneumocystis murina detected in the $\operatorname{Irgm1^{-1}}$ lung by PCR (data not shown). Moreover, similar lesions were found in the lungs, salivary glands, and pancreas of germ-free $\operatorname{Irgm1^{-/}}$ mice (Supplemental Figure 8), confirming that the SS-like tissue phenotype was autonomous from microbiota.

Tissue lesions of Irgm1-1- mice are abolished by Irgm3 deletion. The alternate IRG subfamily $\mathrm{M}$ member, Irgm3, has been proposed to have complex functional cooperativity with Irgm1 in which it coregulates the organellar deposition and activation of other IRG proteins within cells (12). Several of the phenotypes of $\operatorname{Irgm1}^{-/-}$mice reportedly revert upon deletion of Irgm3 (i.e., in $\operatorname{Irgm1^{-/-}} \operatorname{Irgm3}^{-/-}$mice), including defective host defense, macrophage motility, autophagy, and stress hematopoiesis $(8,10)$. It has been proposed that Irgm3 deletion relieves the cytopathic effects of Irgm1 deletion by promoting intracellular clearance/relocalization of aggregated IRG proteins away from lysosomes $(10,12)$. Remarkably, we found that lymphocytic infiltration in lung, salivary glands, lacrimal glands, and pancreas was normalized in $\operatorname{Irgm1^{-1-}} \operatorname{Irgm3}^{-/-}$mice (Figure 4, G-J), indicating that the spontaneous SS-like phenotype of $\operatorname{Irgm1^{-1-}}$ mice is $\operatorname{Irgm} 3$ dependent and likely driven by IRG protein dysregulation.

Irgm1 deletion induces spontaneous IgA-predominant autoimmunity. The lymphocytic infiltration of several exocrine tissues in $\operatorname{Irgm1^{-/}}$ mice was reminiscent of human autoimmune disease, SS in particular. Given this, we tested $\operatorname{Irgm1} 1^{-/}$serum for the presence of anti-nuclear antibodies (ANAs) using HEp-2 cells as substrate, a classical indirect immunofluorescence procedure used in clinical medicine (and mouse models) to screen for autoantibodies targeting nuclear antigens (20). Of interest, although IgG class ANAs were not detected in $\operatorname{Irgm1}^{-/-}$(or WT) serum, an abnormal increase in IgA ANA was evident, yielding nuclear staining in a coarse speckled pattern (Figure $5 \mathrm{~A}$ ).

In patients, a coarse speckled pattern of ANA is specific for autoimmune disease (21). Given this, we used ELISA to quantify specific nucleus-targeting autoantibodies, testing serum from Irgm $1^{-1-}$ mice of 2 ages (8-12 weeks, 1 year) (Figure 5, B and C). Anti-double stranded DNA (anti-dsDNA) is a commonly used confirmatory autoantibody in human autoimmune disease. Anti-Ro and anti-La, ribonucleoproteinspecific autoantibodies commonly found in SS, have been proposed to arise from autoreactive targeting of mucosal epithelia $(1,22)$. Consistent with the ANA finding, anti-dsDNA IgA was dramatically increased in the serum of $\mathrm{Irgm1}^{1^{-1}}$ mice of both ages. By contrast, anti-dsDNA IgG was modestly increased only in young $\operatorname{Irgm} 1^{-/-}$mice and anti-dsDNA IgM was not elevated. Sexual dimorphism was evident for IgA levels of anti-Ro (both Ro-52/tripartite motif-containing protein 21 [Trim21] and Ro-60 antigens) and anti-La, with elevation only in female $\operatorname{Irgm1^{-/}}$ mice, and a dramatic age-dependent further increase in titer (Figure 5C). Anti-Ro IgG and anti-La IgG levels were normal (data not shown).

Autoimmune disorders commonly exhibit systemic induction of cytokines. In SS, excess type I IFNs induce autoantigens and enhance $\mathrm{B}$ cell function and IgA class switching via the IFN-stimulated gene (ISG) BAFF, leading to a vicious cycle $(1,22)$. A wide range of cytokines including BAFF and CXCL13 
A

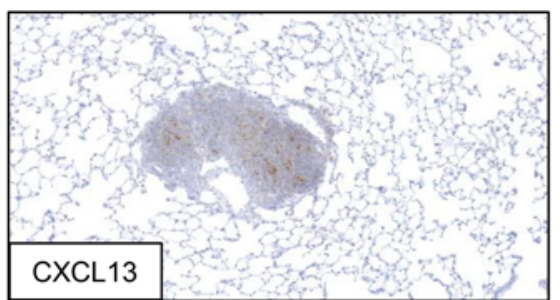

C

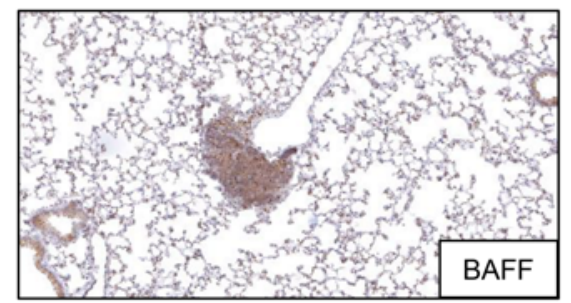

E 1.5

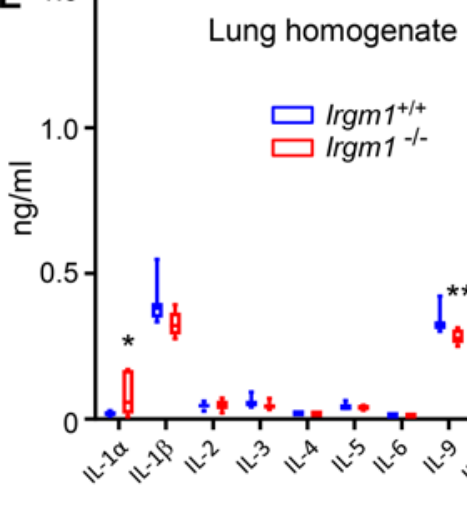

$\mathbf{F}$

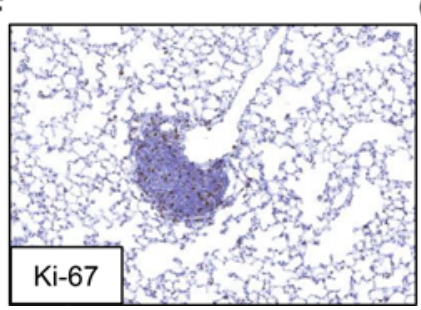

B

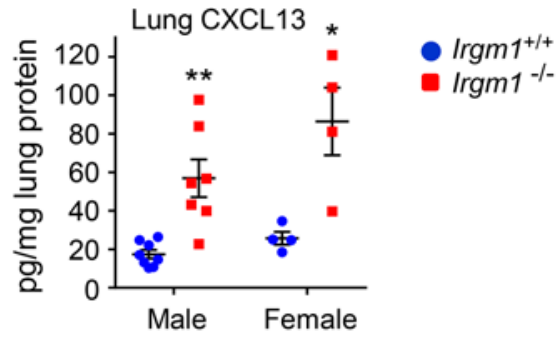

D

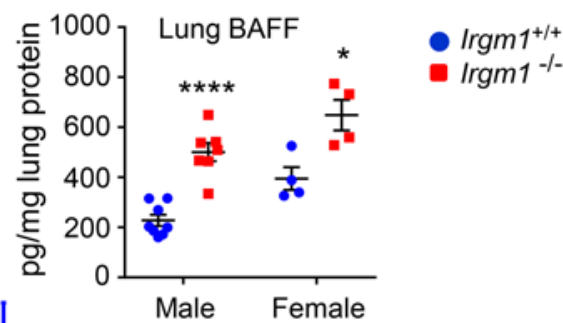

Figure 3. Lung lesions of Irgm1-/- mice display active germinal centers. (A-D) Lungs of naive $/ \mathrm{rgm7^{-/- }}$ mice and controls were evaluated by IHC and ELISA for CXCL13 (A and B) and B cell-activating factor (BAFF) (C and D). Original magnification for panels $\mathbf{A}$ and $\mathbf{C}, \times 20$. For ELISA, 4-8 mice/genotype were used. (E) Cytokines were quantified in lung homogenates from naive Irgm $1^{-1-}$ mice and controls ( $n=7 /$ genotype) using multiplex technology. (F and $\left.\mathbf{C}\right)$ Lung lesions of $/ \mathrm{rgm7}^{-1 /}$ mice were stained by IHC for (F) Ki-67 (original magnification, $\times 20$ ) and (G) peanut agglutinin (PNA; original magnification, $\left.\times 40\right)$. (H) $\mathrm{CD} 45^{+} \mathrm{B} 22 \mathrm{C}^{+} \mathrm{CL7}+\mathrm{CD} 38^{-}$germinal center B cells were quantified in lung digests of naive $\mathrm{lrgm1^{-1- }}$ mice and controls $(n=4-5 /$ genotype). Data are the mean \pm SEM and are representative of at least 3 independent experiments. IHC images are representative of at least $3-4$ mice. ${ }^{*} P<0.05$, ${ }^{* *} P<0.01,{ }^{* *} P<0.001$, ${ }_{* * *}^{* *} P 0.0001$ by unpaired 2-tailed Student's $t$ test.

were elevated in the serum of naive 8- to 12 -week-old $\operatorname{Irgm1^{-1-}}$ mice (Figure 5, D and E). BAFF plus several additional ISGs, including Irf7, Eif2ak2, and $M x 2$ were also upregulated in naive Irgm1 ${ }^{-1-}$ lung tissue (Figure 3D and Figure 6A). Suggesting increased local IFN effect specifically in pulmonary epithelial cells, we found increased expression of Irf7, Eif2ak, Mx2, as well as the ISG Oas1a in epithelial (CD31-CD34$\mathrm{CD} 45-\mathrm{EpCAM}^{+}$) cells sorted from the lungs of naive $\mathrm{Irgm}^{1^{-/}}$mice (Figure 6B and Supplemental Figure 9). By contrast, BAFF (encoded by Tnfsf 136 ) was not elevated, suggesting a nonepithelial local source for this cytokine. Of interest, the SS autoantigen Ro-52 (encoded by Trim21), itself an ISG (23), was also modestly upregulated in hematopoietic $\left(\mathrm{CD} 45^{+}\right)$but not epithelial cells from $\operatorname{Irgm} 1^{-/-}$lungs (Figure 6, B and C). Thus, $\operatorname{Irgm} 1^{-/-}$mice display a pattern of IgA-predominant autoantibody elevation in the context of B cell infiltration into peribronchial regions, in which location BAFF, an IgA class-switch-promoting factor, and Ro-52, a signature SS autoantigen, are both locally increased as part of a larger tissue IFN signature. 
A

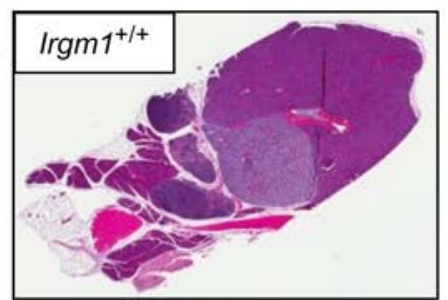

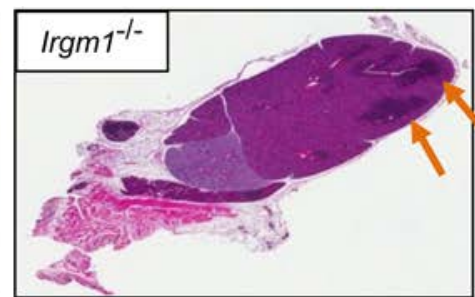

B
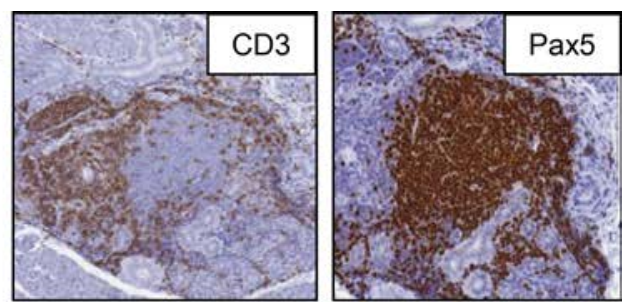

C
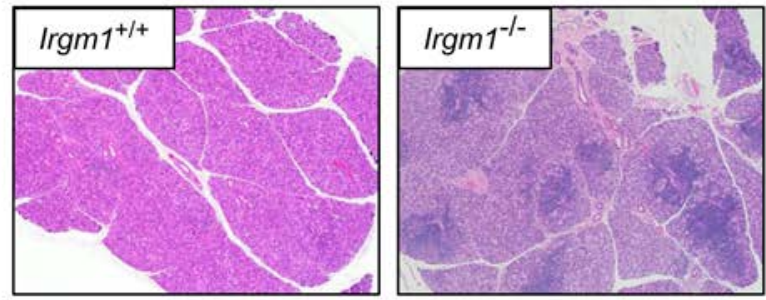

D
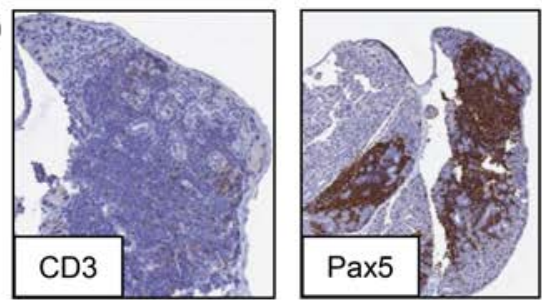

E

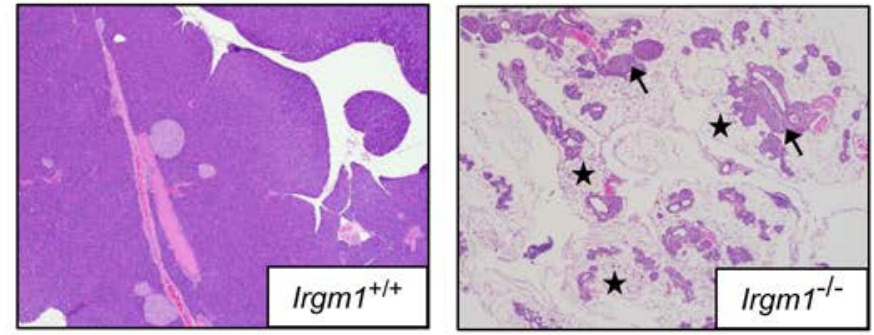

$\mathbf{F}$
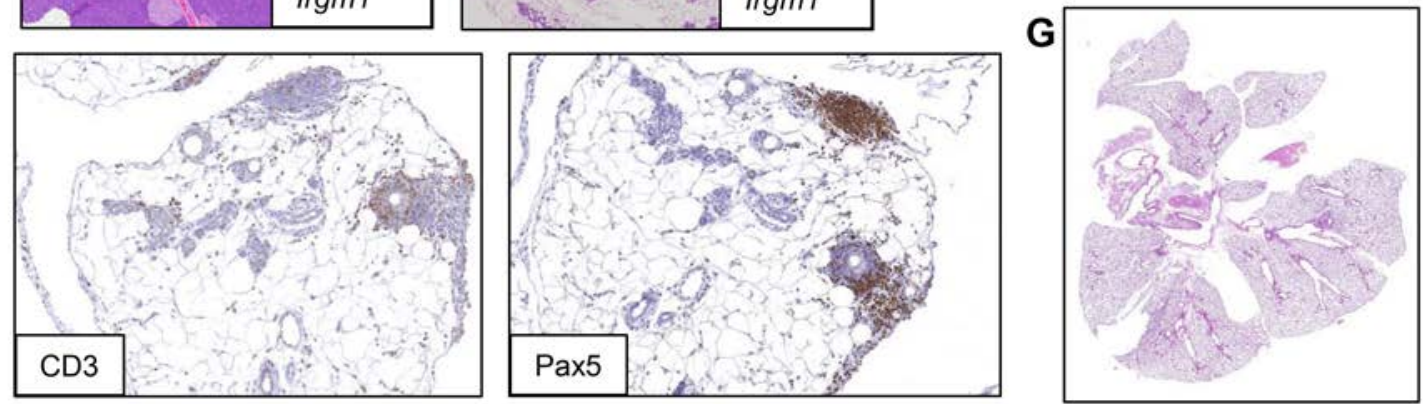

H
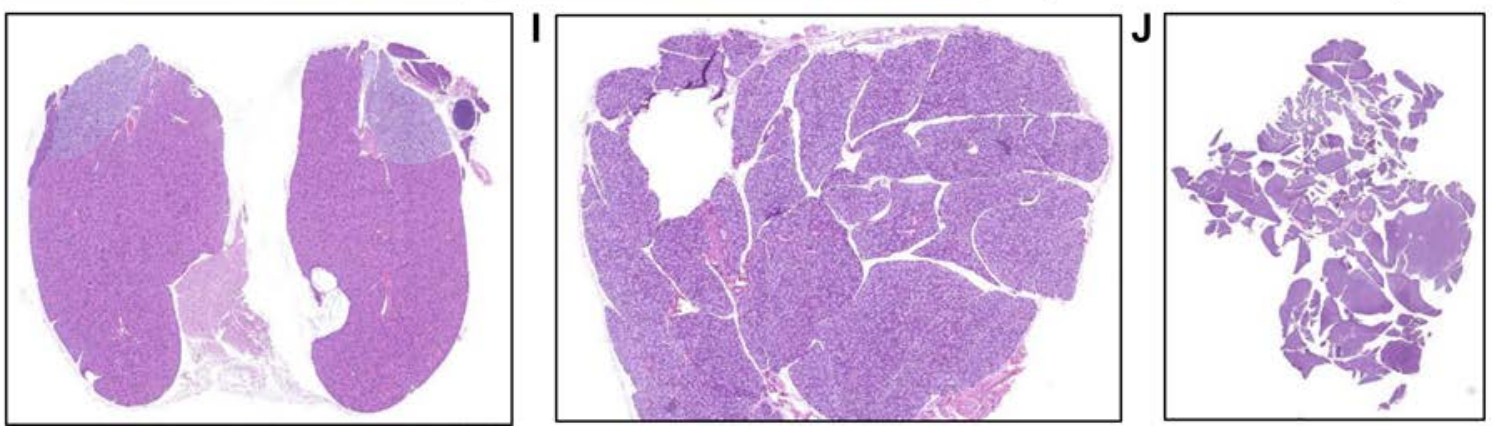

Figure 4. Lymphocytic infiltration of multiple exocrine tissues in $\mathbf{I r g m 1 ^ { - 1 - }}$ mice. (A and B) Submandibular salivary glands from naive $/ \mathrm{rgm} 1^{1 /-}$ mice and controls were stained with hematoxylin and eosin (H\&E; original magnification, $\times 1.4)(\mathbf{A})$, and lymphocytic lesions detected in $/ \mathrm{rgm} 7^{1 /-}$ tissue (shown by arrows in A) were then IHC stained for T cells (CD3) and B cells (Pax5) (original magnification, $\times 40$ ) (B). (C and D) Extraorbital lacrimal glands from naive

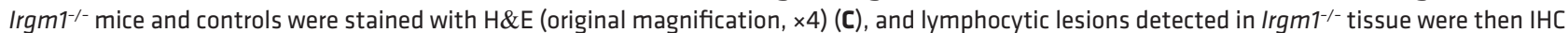
stained for T cells (CD3) and B cells (Pax5) (original magnification, $\times 20$ ) (D). (E and F) Pancreas from naive $/ r g m 1^{-/-}$mice and controls was stained with H\&E (original magnification, $\times 4$ ) (E). Arrows indicate ducts and islets, which are unaffected; asterisks indicate surrounding adipose replacement of exocrine regions. Rare lymphocytic infiltrates in Irgm1 1/- tissue were IHC stained for T cells (CD3) and B cells (Pax5) (original magnification, $\times 20)$ (F). (G-J) Lungs (G), submandibular salivary glands $(\mathbf{H})$, extraorbital lacrimal glands $(\mathbf{I})$, and pancreas (J) were evaluated by H\&E stain in naive $1 \mathrm{rgm} 1^{-1-} \mathrm{Irgm3^{-/- }}$ mice. Original magnification, $\times 0.9$ for $\mathbf{G}$ and $\mathbf{J}, \times 1.4$ for $\mathbf{H}$, and $\times 4$ for $\mathbf{l}$. Tissues in all cases are representative of at least 3-4 mice per genotype.

Irgm1-null lymphocytic lung lesions produce antibody locally. Human autoimmune disorders including SS commonly exhibit polyclonal hyperglobulinemia. Given the reduction in circulating and bone marrow B cells in the face of increased B cells in lung and other tissues of Irgm $1^{-1-}$ mice, it was of interest to define systemic and tissue antibody levels. $\operatorname{Irgm} 1^{-1-}$ mice 8-12 weeks old had a marked increase in serum total IgG 
A

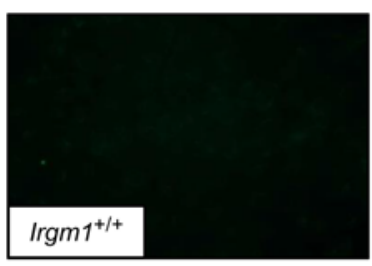

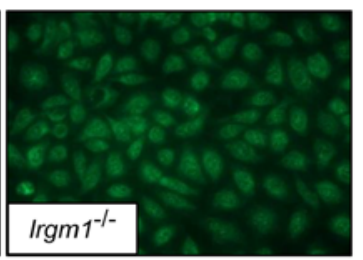
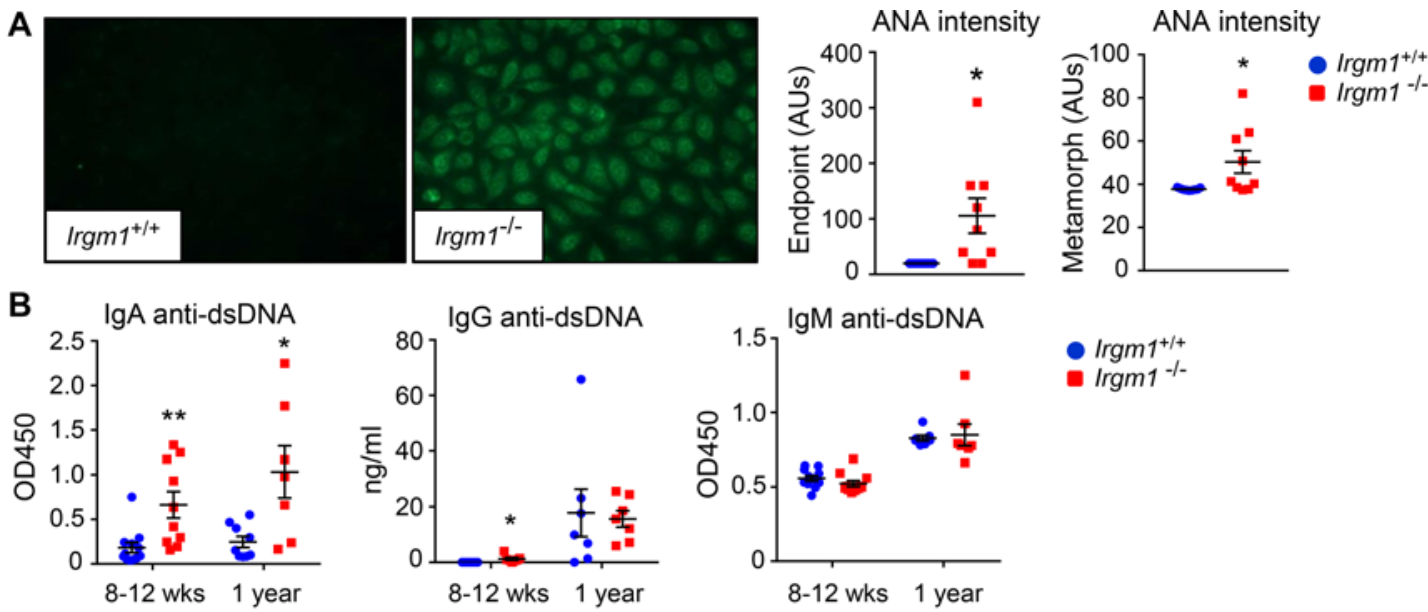

$\operatorname{lrgm} 1^{+/+}$

- $\operatorname{lrgm} 1^{-1-}$
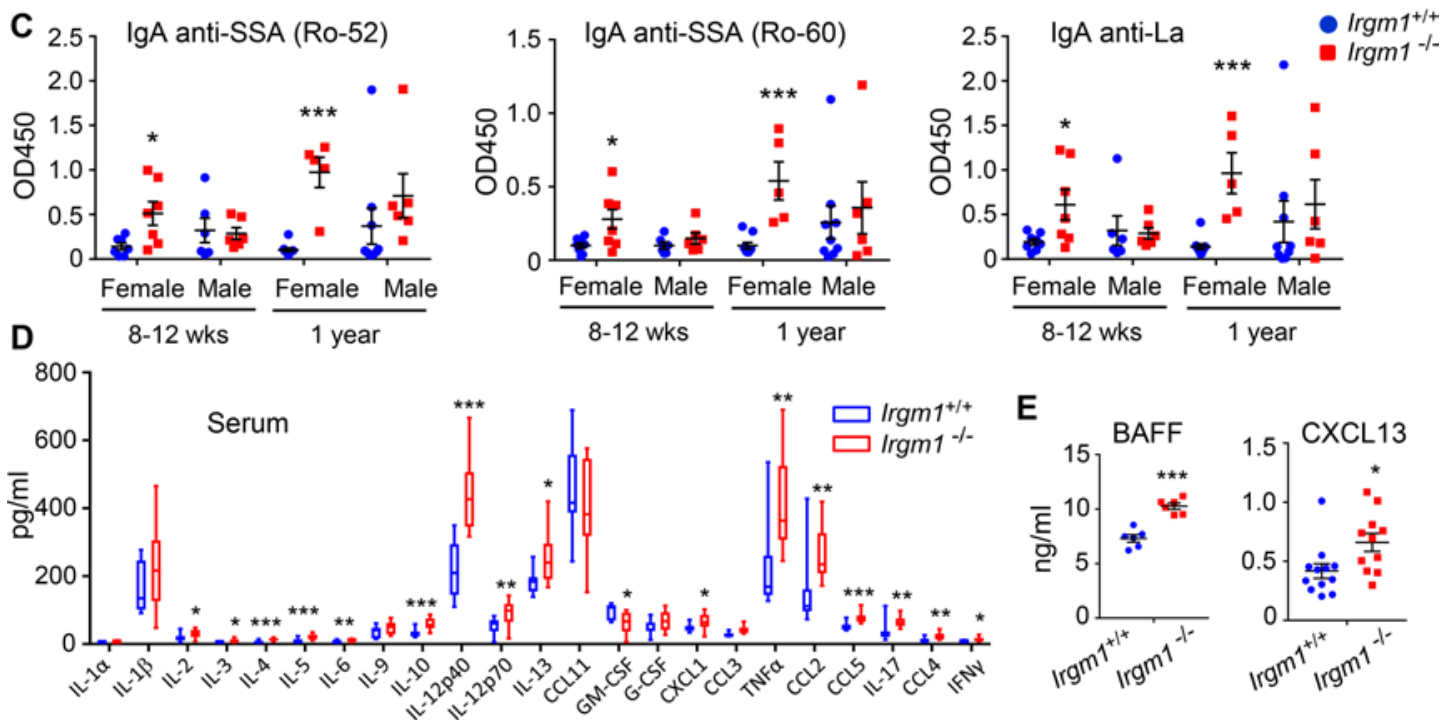

Figure 5. IgA-predominant autoimmunity in naive Irgm1-/- mice. (A) Serum from naive $/ \mathrm{rgm} 1^{-/-}$mice and controls ( $\left.n=9 / g e n o t y p e\right)$ was evaluated for IgAclass anti-nuclear antibodies (ANAs) by indirect immunofluorescence staining of HEp-2 cells. At right, ANA staining was quantified by endpoint (reciprocal of last dilution revealing discernible nuclear staining pattern) and MetaMorph (intensity) analysis (AUs = arbitrary units). (B) IgA, IgG, and IgM anti-double stranded DNA (dsDNA) antibodies were quantified in serum of Irgm1/- mice and controls of 2 ages using ELISA ( $n=7-12 /$ condition). (C) IgA anti-SSA

(Ro-52 and Ro-60) and anti-La antibodies were quantified in male and female mice of 2 ages ( $n=6-10 /$ condition). (D) Cytokines were quantified in serum of Irgm1/-/- mice and controls using multiplex technology ( $n=10-13 /$ genotype). (E) Serum B cell-activating factor (BAFF) and CXCL13 were quantified by ELISA ( $n=6-12$ /genotype). Data are the mean \pm SEM and are representative of at least 3 independent experiments. ${ }^{*} P<0.05,{ }^{*} P<0.01,{ }^{* * *} P<0.001$ by unpaired 2-tailed Student's $t$ test.

and $\operatorname{IgA}$, but not $\operatorname{IgM}$ (Figure 7A). Increased $\operatorname{IgG}$ and, in particular, marked elevation of IgA, was also noted in BALF of naive $\operatorname{Irgm1}^{-1-}$ mice (Figure 7B), whereas the concentration of albumin, a serum protein, was not increased (data not shown). This, together with past reports that airspace IgA derives from local synthesis (24), suggested that the increase in airway immunoglobulin was likely arising, at least in part, from increased local antibody production in the lung. Dimeric IgA made by submucosal B cells is secreted across mucosal epithelia in complex with polymeric immunoglobulin receptor (pIgR) after the latter is proteolytically cleaved into so-called secretory component (SC) (25). Of interest, we found elevated expression of pIgR in $\operatorname{Irgm1}^{-/-}$lungs (Figure 7C). Consistent with increased local release of secretory IgA into the airspace, we found elevated SC in $\operatorname{Irgm1}^{-1-}$ BALF (Figure 7D), as well as increased high molecular weight $(>250 \mathrm{kDa})$ IgA signal on nonreducing immunoblot of $\operatorname{Irgm}^{1^{-1}}$ BALF (Figure 7E).

IHC of the $\operatorname{Irgm1^{-/-}}$ lung confirmed that B cells in the center of the follicular lesions were predominantly $\mathrm{IgM}^{+}$, but that $\mathrm{IgG}^{+}$and $\mathrm{IgA}^{+}$cells were also present (Figure $7 \mathrm{~F}$ ), suggesting that class switch recombination was occurring locally. In order to quantify local antibody release from B cells in various tissues, ELISPOT was performed (Figure 7, G and H). In bone marrow and spleen of $\operatorname{Irgm}^{-1^{--}}$mice, no change in frequency 
A
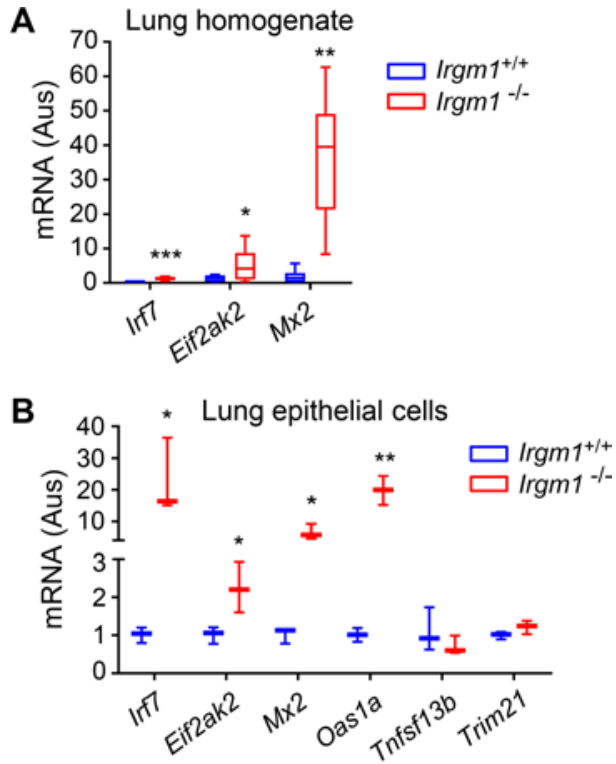

C

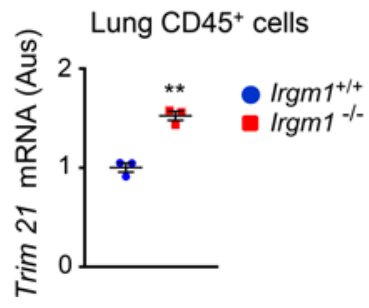

Figure 6. Spontaneous induction of interferon-stimulated genes in the $\operatorname{Irgm1^{-/-}}$ lung. mRNA expression (normalized to GAPDH) of the indicated targets was quantified in whole lung (A), pulmonary epithelial (CD45-CD31-CD34-EpCAM+) cells sorted by FACS (B), and pulmonary hematopoietic (CD45') cells purified by column (C) from naive $\mathrm{Irgm}^{1 /-}$ and $\mathrm{Irgm} 1^{+/+}$mice $(n=3-7 /$ genotype). Data are the mean \pm SEM and are representative of at least 3 independent experiments. ${ }^{*} P<$ $0.05,{ }^{* *} P<0.01,{ }^{* *} P<0.001$ by unpaired 2 -tailed Student's $t$ test.

or absolute number of IgG antibody-secreting cells (ASCs) was detected compared with WT counterparts. By contrast, although not statistically significant, a reciprocal decrease in IgM and increase in IgA ASCs was detected in both tissues, suggesting increased IgA class switching. In the lung, ASCs of all 3 classes were markedly increased, suggesting that the lung, and almost certainly the B cell-rich foci specifically, is a site of robust antibody production in Irgm1-deleted animals. Consistent with increased local antibody production, total spot area (i.e., integrated $\mathrm{Ig}^{+}$area surrounding all ASCs) on ELISPOT was markedly increased for all 3 antibody classes in lung tissue (Supplemental Figure 10). In addition, ELISA of saline-perfused Irgm $1^{-1-}$ lung tissue confirmed increased IgM, IgG, and IgA, with most marked elevation in the latter (Figure 7I).

Irgm1-null lung overproduces T15-idiotype anti-PC IgA. Natural antibodies are germlineencoded, innate (i.e., spontaneous), polyreactive immunoglobulins, largely produced by B1a B cells, that are thought to have been evolutionarily selected in response to important antigens (26). Among natural antibodies are the well-studied T15-idiotype anti-PC autoantibodies, which are reactive against oxidized phospholipid (i.e., oxidized 1-palmitoyl-2-arachidonoyl-sn-phosphatidylcholine) in apoptotic cells, as well as against PC exposed on the surface of S. pneumoniae (14). Given that B1a cells and anti-PC play complex roles in autoimmunity $(27,28)$ and accumulate in the lung in chronic inflammatory conditions (29), we surveyed for dysregulated levels of anti-PC immunoglobulins in the lung and other tissues of $\operatorname{Irgm1^{-/-}}$ mice.

$\operatorname{Irgm} 1^{-1-}$ mice had a reciprocal decrease in PC-specific IgM and increase in PC-specific IgA in the serum, whereas PC-specific IgG levels were equivalent to WT (Figure 8A), together suggesting increased IgA class switching, perhaps due to increased anti-PC production at a mucosal site. ELISPOT revealed an increase in frequency and number of anti-PC IgA ASCs in Irgm1 $1^{-1-}$ lung tissue (Figure 8B). This was associated with increased PC-specific IgA in BALF of Irgm $1^{-/-}$mice (Figure 8C). An increase in T15-idiotype anti-PC IgA was also detected in the BALF, lung tissue, and serum of $\operatorname{Irgm1^{-1-}}$ mice (Figure 8D). TC68 and AB1-2 are anti-T15 idiotype antibodies; the former detects the $\mathrm{V}_{\mathrm{H}} \mathrm{S} 107.1$ region of the T15 idiotype, and the latter, the CDR3 region (30). Consistent with increased local production of T15 IgA in the $\operatorname{Irgm1^{-/-}}$ lung, we detected increased frequency and numbers of $\mathrm{B} 220^{+} \mathrm{PC}-\mathrm{BSA}^{+} \mathrm{TC} 68^{+} \mathrm{AB} 1-2^{+}$cells in lungs of $\operatorname{Irgm1^{-/}}$ mice (Figure 8E and Supplemental Figure 11). These findings suggest that, even in the face of reduced circulating and bone marrow B cells, Irgm1 deletion favors trafficking of B1a cells to the lung, at which site local conditions including BAFF overexpression may favor both enhanced survival and IgA class switching of T15-idiotype cells.

Augmented innate anti-Pneumococcal host defense in the Irgm1-null lung. Exogenous treatment with T15-idiotype antibody improves survival in mice infected by $S$. pneumoniae, likely due to enhanced PCtargeted binding (14). Given this, we speculated that the local increase in native airway T15-idiotype antibody in $\operatorname{Irgm1} 1^{-1-}$ mice might be associated with augmented natural host defense. To test this, $\operatorname{Irgm1^{-1}}$ mice and controls were infected intratracheally with PKH26-labeled $S$. pneumoniae. Analysis of BALF collected 30 minutes after infection revealed increased IgM and IgA opsonization of S. pneumoniae in the $\operatorname{Irgm} 1^{-1-}$ airway (i.e., an increased percentage of $\operatorname{IgM}^{+}$and $\operatorname{IgA}^{+} \mathrm{PKH}_{26} 6^{+}$bacteria) (Figure 9, A and B). This augmented recognition by natural antibody was associated with enhanced clearance of $S$. pneumoniae from the lung 48 hours after infection (Figure 9C), and also with improved survival of $\operatorname{Irgm1^{-/-}}$ mice following $S$. pneumoniae infection (Figure 9D). With $S$. pneumoniae, we thus extend the purview of $\operatorname{Irgm} 1$ in host defense to extracellular bacteria, showing in this case, contrary to that of intracellular pathogens, that Irgm 1 deletion augments successful pathogen clearance. Taken together, these findings suggest that Irgm1 deletion confers a coordinate disinhibition of autoimmunity and innate immunity at selected mucosal sites, with increased local production of IgA-predominant antibodies targeting evolutionarily conserved lipid moieties common to host and pathogens. 

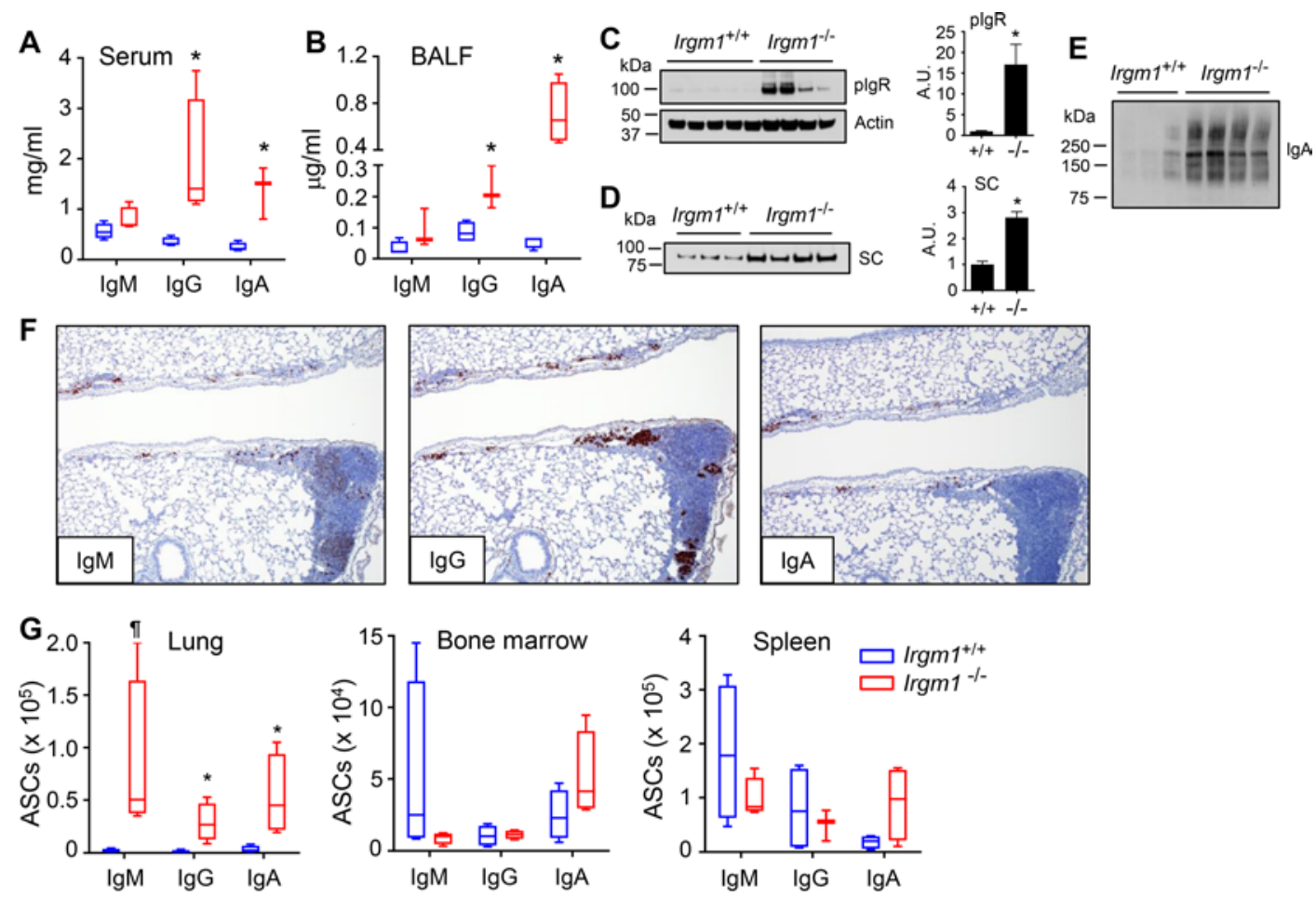

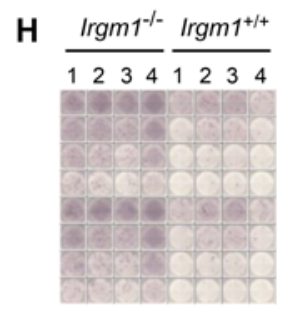

$\lg M$

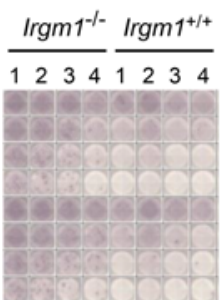

$\lg G$

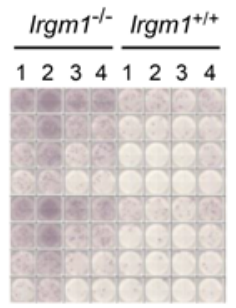

$\lg A$

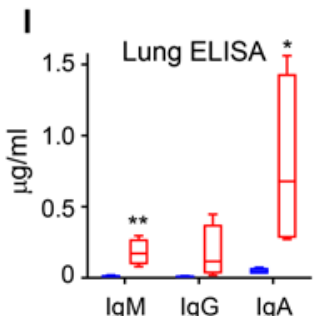

Figure 7. Antibody production by the Irgm1-/- lung. (A and B) IgM, IgG, and IgA were quantified by ELISA in serum (A) and bronchoalveolar lavage fluid (BALF) (B) of naive Irgm1/-- mice and controls ( $n=4 /$ genotype). (C and D) Polymeric lg receptor (plgR) was detected by immunoblot in equal protein aliquots of lung homogenate (C) ( $n=4-5 /$ genotype) and BALF, i.e., as cleaved secretory component (SC) (D) ( $n=3-4 /$ genotype), from independent mice as shown. Actin serves as a loading control. Densitometry of the corresponding blots is shown at right (AU = arbitrary unit). (E) IgA was detected by immunoblot under nonreducing conditions in BALF of naive mice as shown. $n=3-4$ /genotype. (F) Immunohistochemically stained sections of naive Irgm1 $1^{-/-}$lungs showing staining for IgM, IgG, and IgA in lymphocytic infiltrates. Original magnification, $\times 10$. (G and $\mathbf{H}$ ) Antibody-secreting cells (ASCs) of the 3 immunoglobulin classes shown were quantified in lung, bone marrow, and spleen of naive $/ \mathrm{rgm} \mathrm{Fr}^{-/-}$mice and controls using ELISPOT. In $\mathbf{H}$ the corresponding serial dilution ELISPOT plates for lung are depicted for $n=4$ mice/genotype. (I) IgM, IgG, and IgA were quantified by ELISA in homogenates of perfused lungs from $\mathrm{lrgm} 1^{-1-}$ mice and controls $\left(n=4 /\right.$ genotype). Data are the mean \pm SEM and are representative of at least 3 independent experiments. ${ }^{~} P=0.08$, ${ }^{*} P<0.05,{ }^{* *} P<0.01,{ }^{* * *} P<0.001$ by unpaired 2 -tailed Student's $t$ test.

\section{Discussion}

Primary SS is the second most common autoimmune disease, with an estimated prevalence of 0.9-6 per 1,000 individuals, involving a 9:1 female predominance (1). Roles have been identified for IFNs, CXCL13, BAFF, and B cells, all of which are proposed to impinge upon epithelial cells, leading to a vicious cycle of autoantibody generation (1). Autoantigens such as Ro externalized on apoptotic cellular blebs may trigger autoantibody production and germinal center formation by infiltrating $B$ cells at mucosal sites (22). While mouse models have been developed that recapitulate features of $\mathrm{SS}$, several of these including (NZB/NZW)F $\mathrm{F}_{1}, \mathrm{MRL} / \mathrm{lp} r$, BAFF-transgenic, IL-12-transgenic, IL-14 $\alpha-$ transgenic, and TGF- $\beta 1^{-/}$mice are dominated by multiorgan involvement in an SLE-like pattern (i.e., glomerulonephritis, skin disease, lymphadenopathy), while others are confounded by comorbidities such as diabetes mellitus (2). Thus, many questions remain about the disease mechanisms specific to SS pathogenesis, and there is a need for additional, well-defined murine models that faithfully mimic the human disorder. 

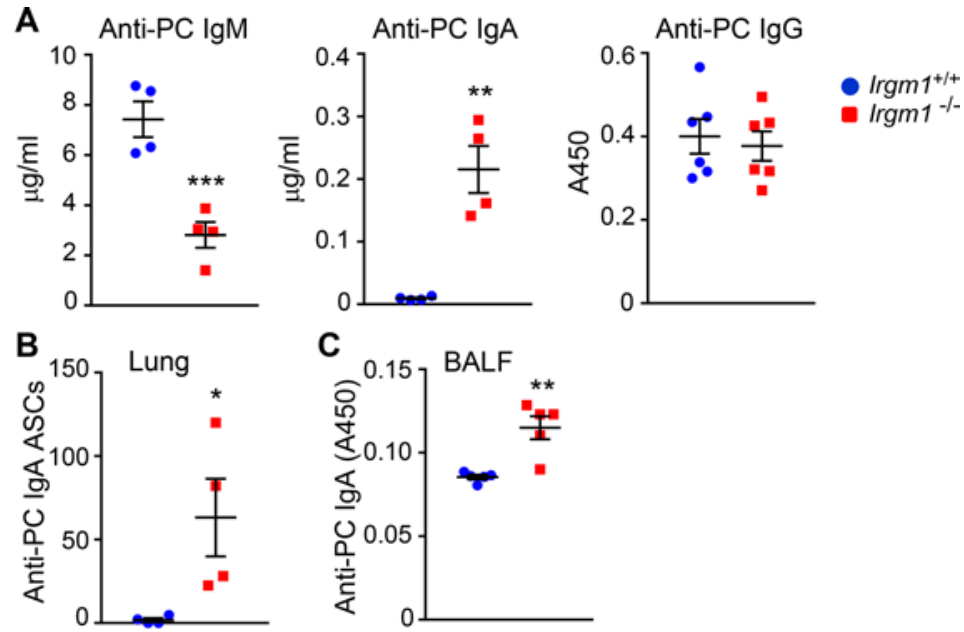

C
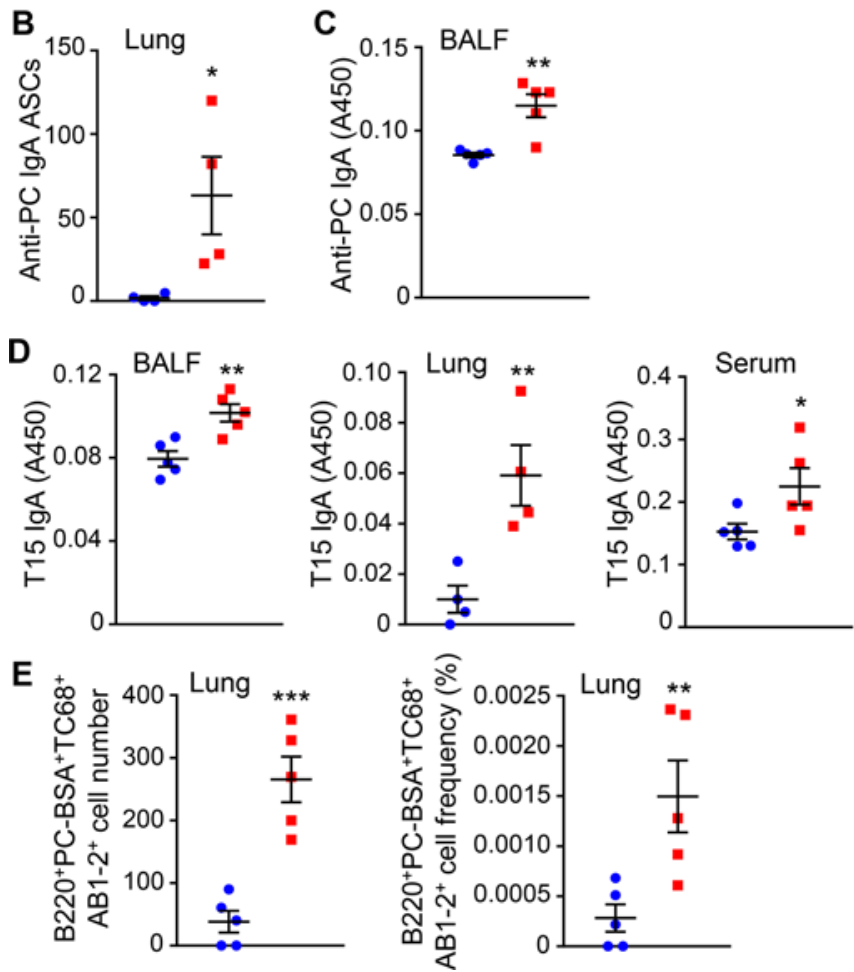

Figure 8. Production of T15-idiotype anti-phosphorylcholine IgA in the Irgm1-/- lung. (A) Anti-phosphorylcholine (anti-PC) IgM, IgA, and IgG was quantified by ELISA in serum of naive $\mathrm{Irgm1^{-/- }}$ mice and controls. (B) Anti-PC IgA antibody-secreting cells (ASCs) were quantified in perfused lung of naive $/ \mathrm{rgm}^{-/-}$mice and controls by ELISPOT. Data were verified by BSA negative control run in parallel and background subtracted. (C) Anti-PC IgA was quantified by ELISA in bronchoalveolar lavage fluid (BALF) of naive $\mathrm{Irgm1}^{-1-}$ mice and controls. (D) T15-idiotype IgA was quantified by ELISA in BALF, lung, and serum of naive $\mathrm{Irgm1}^{-/-}$mice and controls. (E) T15-idiotype $B$ cells were quantified by flow cytometry in lung of naive $1 \mathrm{rgm}^{-1-}$ mice and controls. Data are the mean \pm SEM and are representative of at least 3 independent experiments involving $n=4-6 /$ genotype. ${ }^{*} P<$ $0.05,{ }^{*} P<0.01,{ }^{* *} P<0.001$ by unpaired 2 -tailed Student's $t$ test.

Here, we report for the first time to our knowledge that $\operatorname{Irgm} 1^{-/-}$mice spontaneously display several features reminiscent of SS by approximately 8 weeks of age, including lymphocytic infiltration of lacrimal and salivary glands, hyperglobulinemia, and elevated BAFF, ANA, and anti-Ro and -La antibodies. We found some evidence for gender dimorphism, in particular, elevated anti-Ro and -La autoantibodies in females. Associated with these hallmark tissue lesions was selective atrophy of the exocrine pancreas consistent with autoimmune pancreatitis, marked expansion of peribronchiolar lymphoid tissue, and, in a subset of mice, lesions potentially consistent with autoimmune hepatitis. All of these are tissue complications that have been associated with human SS $(22,31)$. In addition to representing a novel model of SS with extraglandular manifestations, the $\operatorname{Irgm1^{-/}}$ mouse may thus also facilitate new molecular insights into BALT and autoimmune pancreatitis.

Of interest, the $\operatorname{Irgm1^{-1-}}$ mouse exhibits increased serum IgG and IgA, but normal IgM. Among antibody-producing tissues surveyed, bone marrow and spleen of the $\operatorname{Irgm~}^{1^{-1}}$ mouse had normal/reduced IgM/IgG ASCs but increased IgA ASCs, whereas lung exhibited a dramatic increase in ASCs for all 3 isotypes. Collectively, these findings suggest increased IgA class switching in $\operatorname{Irgm} 1^{-1-}$ tissues, and also the interesting possibility that the prominent lung lesions may serve as a significant source of systemic immunoglobulin in the $\operatorname{Irgm1^{-/}}$ mouse. Increased autoantibody levels in the $\operatorname{Irgm1^{-/}}$ mouse were restricted to the IgA class, perhaps pointing to an affected mucosal site as a source of origin. Consistent with this, we confirmed the lung as a source of T15-idiotype anti-PC IgA, and identified increased pulmonary B1a cells as a likely source (26). Whereas several prior publications have identified macrophage and $\mathrm{T}$ cell abnormalities in the setting of Irgm1 deletion $(9,15,16)$, we are unaware of past reports of B cell dysregulation in $\operatorname{Irgm1}^{1^{-1-}}$ mice.

B1a cells have previously been implicated in autoimmunity (28), in which context they may be attracted to affected target organs by CXCL13 (32), a chemokine we found to be elevated in the follicular lesions of the $\operatorname{Irgm1^{-/}}$ lung (Figure 3A), as well as in the salivary and lacrimal gland lesions of $\operatorname{Irgm1^{-/}}$ mice (data not shown). CXCL13 is reportedly required for B1 cell production of T15 anti-PC antibodies (33). B1a cells have been identified as major sources of $\operatorname{IgA}$ in vivo (34), and also shown to class switch to IgA preferentially, especially in response to BAFF (19), which we also found to be increased in Irgm $1^{-1-}$ lung lesions. Aberrant expression of select cytokines may thus underlie abnormal homing of B1a cells to the $\operatorname{Irgm1^{-/-}}$ lung as well as programming of B1a cells at that site to release IgA class natural antibodies. Suggesting human relevance, BAFF and CXCL13 are also elevated in human SS and $80 \%$ or more of primary SS patients have elevated anti-Ro and -La IgA $(1,35,36)$.

Irgm1 is an IFN-stimulated p47 GTPase that has been proposed to support host defense against intracellular pathogens by promoting autophagy and mitochondrial homeostasis $(7,8,10)$. It is widely expressed in murine immune cells, including $\mathrm{B} 1$ and $\mathrm{B} 2$ cells, $\mathrm{CD} 4^{+} \mathrm{T}$ cells, $\mathrm{CD} 8^{+} \mathrm{T}$ cells, conventional 
A
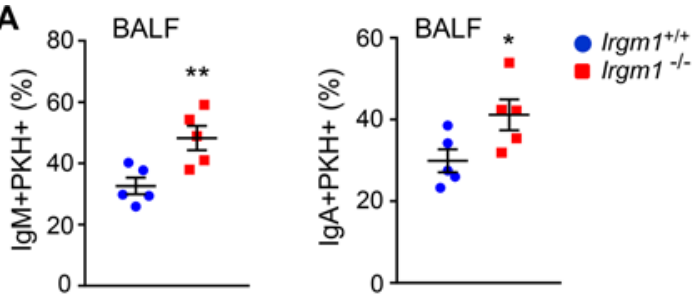

B Uninfected

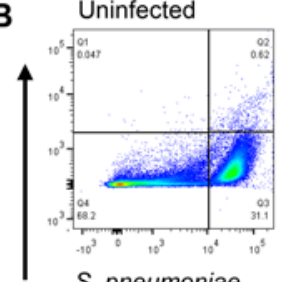

S. pneumoniae
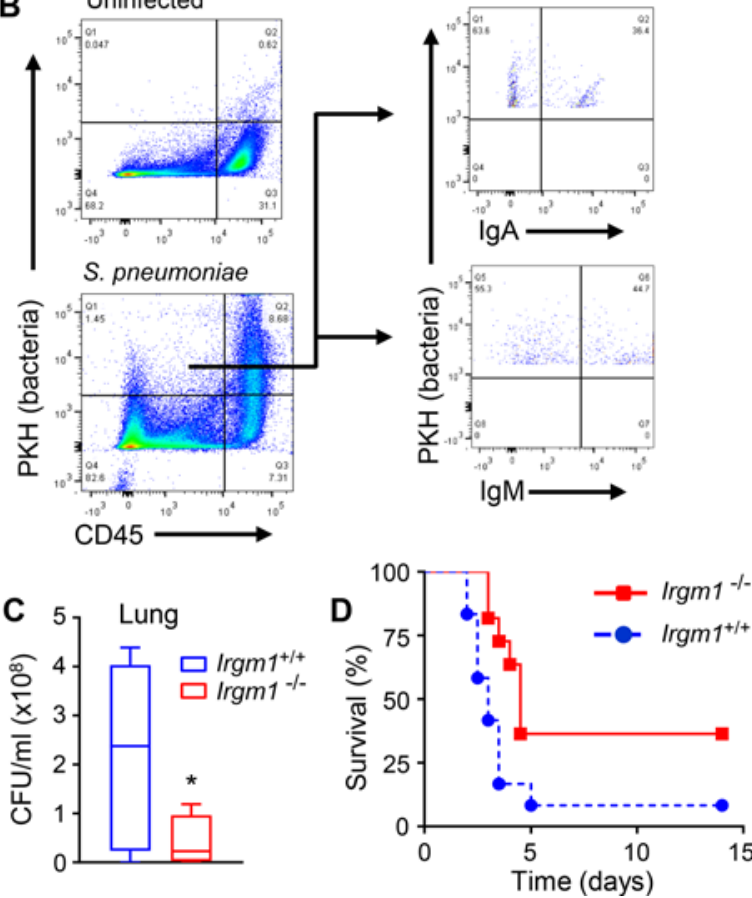

Figure 9. Enhanced anti-Pneumococcal host defense in $\mathbf{I r g m 1 ^ { - 1 - }}$ mice. (A and B) Mice ( $n=5$ /genotype) were infected in the lungs with PKH26-labeled Streptococcus pneumoniae. Thirty minutes after infection, the percentage of IgM- and IgAopsonized cell-free $\left(\mathrm{CD}^{-} 5^{-}\right)$extracellular bacteria was quantified by flow cytometry The gating strategy is shown in B. (C) Lung bacterial colony-forming units (CFUs) were quantified 48 hours after infection of $/ \mathrm{rgml}^{-1-}$ mice and controls with $\mathrm{S}$. pneumoniae ( $n=6-7 /$ genotype). (D) Survival was monitored in $/ \mathrm{rgm} 1^{-1-}$ mice and controls ( $n=10$ /genotype) following lung infection with S. pneumoniae. Data are the mean \pm SEM and are representative of at least $2-3$ independent experiments. ${ }^{*} P<0.05,{ }^{* *} P<0.01$ by unpaired 2 -tailed Student's $t$ test. Survival was evaluated by log-rank test $(P<0.05)$.

and plasmacytoid DCs (pDCs), and macrophages (www.immgen.org). Recent reports have identified a link between an IRGM polymorphism associated with reduced gene expression and Crohn's disease (13). These findings have suggested that the human homolog of $\operatorname{Irgm} 1$ may suppress spontaneous mucosal immunity against self and/or microbiota. Somewhat at odds with this are recent reports that $\operatorname{Irgm} 1$ deletion is protective against experimentally enforced autoimmunity (37). However, experimental autoimmune encephalomyelitis and similar artificial models, involving vaccination and acute challenge with autoantigens, almost certainly diverge from the pathogenesis of native autoimmune disease.

Irgm $1^{-1-}$ leukocytes reportedly display enhanced TLR signaling through the adaptor protein myeloid differentiation program protein 88 (MyD88) (38). In other settings, MyD88 has been shown to support TLR7-dependent induction of type I IFNs by pDCs (39), BAFF production by and signaling in B cells $(40,41)$, as well as several B cell functions including autoantibody production (42). Hyperactivated MyD88-dependent signaling in DCs is sufficient to cause autoimmune disease in mice (20). Taking these results together, we thus propose that enhanced MyD88-dependent

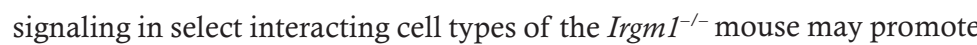
an axis of IFN overproduction, downstream BAFF induction, and BAFF hypersensitivity, leading to autoimmunity. Using ELISA, we found only very low levels of IFN- $\alpha$ in BALF of $\operatorname{Irgm1}^{-1 /}$ (and $\operatorname{Irgm} 1^{+/+}$) mice that were near the limit of detection (data not shown); however, a recent report using a highly sensitive viral suppression bioassay did confirm elevated type I IFNs in serum of $\operatorname{Irgm1^{-/-}}$ mice (43). Given that elevated type I IFNs reportedly drive increased epithelial cell death and turnover in the pancreas and salivary gland of $\operatorname{Irgm1^{-/-}}$ mice (43), we further speculate that IFN overproduction may target autoreactive $\mathrm{B}$ cells to the $\mathrm{Irgm}^{1^{-/}}$epithelium. Interestingly, MyD88 may possibly contribute to autoimmune targeting of $\operatorname{Irgm1} 1^{-/-}$mucosa given that it reportedly underlies autoimmune targeting of the lung and other mucosal surfaces in Foxp3-deficient mice (44).

As has been reported for the host defense defects of the $\operatorname{Irgm} 1^{-1-}$ mouse (12), we found that the SS-like phenotype was abolished by Irgm3 deletion. Irgm3 deletion has been proposed to rescue cellautonomous defects in Irgm1-null cells by restoring dysregulated subcellular localization and activation of IRG proteins (12). Our results thus suggest that Irgm1-disinhibited IRGs in one or more interacting cell types may be the proximal cause of autoimmune pathogenesis. This could arise, for example, from damage-associated molecular patterns released by Irgm1-deficient cells inducing IFN production in $\mathrm{pDC}$ or other cells through TLR/MyD88 signaling. Identification of the Irgm1-deficient cell types that drive the in vivo SS-like phenotype will have to await generation of tissue conditional $\operatorname{Irgm1^{-/-}}$ mice.

Irgm $1^{-1-}$ cells have deficient autophagic flux (11). However, mice with deficient canonical autophagy do not display spontaneous autoimmune disease (45). Moreover, B cell autophagy is required for the SLE-like phenotypes of TLR7-transgenic mice and $\mathrm{Lpr}$ mice $(46,47)$, as well as for differentiation and survival of plasma cells (48). There is evidence for increased autophagic flux in T cells in human SLE and murine SLE models (49). We thus speculate that deficient autophagy is unlikely to be the underlying mechanism of spontaneous autoimmunity in $\mathrm{Irgm}^{-/-}$mice, and may possibly mitigate against it. 
Future studies will be required to better define the mechanisms underlying the enhanced anti-Pneumococcal host defense of the $\operatorname{Irgm} 1^{-1-}$ lung. The degree to which locally produced secretory IgA versus $\operatorname{IgM}$ or other antibody isotypes contribute is uncertain. T cells and other cells of the local BALT-like aggregates may also collaborate in the immune response. Finally, the possibility also remains that altered recruitment of circulating immune cells (e.g., neutrophils) to the infected $\operatorname{Irgm1^{-1-}}$ airspace may contribute to enhanced bacterial clearance. Detailed immune profiling of the infected lung, as well as cell-specific Irgm1 deletion may ultimately be required to properly dissect the role(s) of Irgm1 in this context.

Taken together, our studies identify $\operatorname{Irgm1}$ as a unifying regulator of self- and other-directed immune responses at selected mucosal sites. Phosphatidylcholine is the predominant phospholipid of pulmonary surfactant, in which location it has remarkably high concentrations not found elsewhere in vivo (50). It is intriguing to consider that this PC-headgroup phospholipid, perhaps oxidized by the inflammatory milieu of the $\operatorname{Irgm1^{-1}}$ lung, may serve as the inciting autoantigen for local expansion of T15-idiotype B cells in the lung. We propose that IRGM polymorphisms should be examined for their possible association with BALT, $\mathrm{B}$ cell homeostasis, and autoimmune disease in humans, and also that investigation of IRGM as a potential therapeutic target in autoimmunity and pulmonary host defense is warranted.

\section{Methods}

Mice. Irgm1-deficient mice have been described (9) and were crossed more than 8 generations onto the C57BL/ 6 background. Age- and gender-matched WT littermate controls were used. Germ-free mice were housed in sterile flexible film Trexler isolators with sterilized bedding and sterilized chow and water (ad libitum), within the National Gnotobiotic Rodent Resource Center, UNC/Chapel Hill. Sterility was confirmed on a monthly basis by fecal Gram stain, aerobic and anaerobic cultures, and 16S rRNA PCR analysis of feces and bedding. $\operatorname{Irgm1^{-1-}} \operatorname{Irgm3^{-1-}}$ mice were obtained from G.A. Taylor.

Flow cytometry. Lung tissues were perfused, digested, and processed to single-cell suspension as previously reported (51). Cells were blocked with anti-mouse CD16/32 (Biolegend, 101302) and stained for 30 minutes with the following anti-mouse antibodies for individual plots: CD45-eFluor 450 (eBiosciences, 48-0451-82); CD3e PE Cy7 (Biolegend, 100320); CD19-FITC (Biolegend, 115507); CD45.2-PE (Biolegend, 109808); CD4-FITC (eBiosciences, 11-0041-81); CD8a APC (Biolegend, 100711); CD45R/B220-eFluor 450 (eBiosciences, 48-0452-82); CD5 PE (Biolegend, 100608); CD11b V500 (BD Horizon, 562128); IgM APC (Biolegend, 406515); CD43 PerCP-Cy5.5 clone S7 (BD Biosciences, 562865); CD3-AF488 (Biolegend, 100321); IgM-PE (Biolegend, 406508); CD43 clone 1B11 APC/Cy7 (Biolegend, 121220); GL7-FITC (Biolegend, 144603); CD38 PE (Biolegend, 250505); IgA-FITC (Southern Biotech, 1165-02); and IgM AF647 (Life Technologies, A21238). S107 (PC-specific IgA) cells, provided by the Kearney laboratory, were used as a positive control. PC-BSA AF488 was generated with the Alexa Fluor 488 Monoclonal Antibody Labeling kit (Invitrogen). Purified monoclonal antibodies for TC68 Pacific Blue and AB1-2 AF555 were provided by the Kearney laboratory. PC-BSA AF488 was generated with the Alexa Fluor 488 Monoclonal Antibody Labeling kit (Invitrogen). CD45R/B220 PerCP was from BD Biosciences (561086). All flow cytometric analyses were performed on the LSR II (BD Biosciences) and analyzed using FACSDiva (BD Biosciences) and FlowJo software (Tree Star).

Clinical blood assays. Blood cell counts were analyzed using the HEMAVET 1700 hematology analyzer (Drew Scientific, Inc.). Manual leukocyte differential counts were performed, and smear estimates were used to confirm values. Serum amylase was measured with an Olympus AU400e (Beckman Coulter) using reagents from the manufacturer.

Immunoglobin and albumin ELISAs. Mouse IgA, IgM, IgG, and albumin ELISA quantitation sets (Bethyl Laboratories) were used with the following dilutions: serum 1:10,000 (IgA, IgM) or 1:50,000 (IgG); tissue ELISAs neat or 1:4; BALF (immunoglobulins) 1:2; and BALF (albumin) 1:1,000.

ANA assay. An indirect fluorescent antibody assay utilizing HEp-2 cells as a substrate (MBL Bion) following the manufacturer's specifications used mouse serum at an initial concentration of 1:40 for screening and then diluted up to 1:640 for endpoint analysis. FITC-conjugated goat anti-mouse IgG ( $\gamma$-chain specific), $\operatorname{IgM}$ ( $\mu$-chain specific), or IgA ( $\alpha$-chain specific) (Sigma-Aldrich) was added to wells at 1:200 in PBS. For MetaMorph (Molecular Devices) analysis, negative controls (PBS) were used to determine a threshold of 35 and most of the data had intensities of 38-39.

Autoantibody ELISAs. Mouse anti-SSB (La), anti-SSA (Ro-52), and anti-SSA (Ro-60) ELISAs (Signosis) were used per manufacturer's specifications for IgG detection, and utilizing HRP-conjugated IgA (Bethyl 
Laboratories) at a dilution of 1:1,000. Serum samples were diluted 1:50. For anti-dsDNA ELISA, calf thymus DNA (Sigma-Aldrich) stock was resuspended at $2 \mathrm{mg} / \mathrm{ml}$ in $1 \times \mathrm{SSC}$ buffer overnight at $4^{\circ} \mathrm{C}$, sheared the next day by running through an 18-gauge needle, and then diluted to $1 \mu \mathrm{g} / \mathrm{ml}$ in carbonate buffer for coating of plates overnight at $4^{\circ} \mathrm{C}$. The next day, wells were washed with $0.5 \times \mathrm{PBS} / 0.5 \% \mathrm{BSA}$, and blocked with PBS/3\% BSA (room temperature, 2 hours). Serum samples (1:50 dilution) and anti-dsDNA antibody (Abcam, clone HYB331-01) used for standard for IgG isotype were diluted using $0.5 \times \mathrm{PBS} / 0.5 \%$ BSA buffer, and plates were incubated at $37^{\circ} \mathrm{C}$ for 1 hour, and then $4^{\circ} \mathrm{C}$ for 1 hour. After washing, HRPconjugated anti-mouse IgG, IgA, or IgM (Bethyl Laboratories) was added to the plate at 1:5,000 dilution in $0.5 \times \mathrm{PBS} / 0.5 \% \mathrm{BSA}$ and wells were incubated at room temperature for 1 hour. Wells were then washed with $0.5 \times \mathrm{PBS} / 0.5 \%$ BSA, developed with TMB substrate (Biolegend), stopped with $2 \mathrm{~N} \mathrm{H}_{2} \mathrm{SO}_{4}$, and read at OD450 (Bio-Tek Synergy 2 microplate reader).

Quantitative RT-PCR. Total RNA was extracted from cells and tissues with RNeasy kits (QIAGEN) using the manufacturer's protocol. For tissue-extracted RNA, tissues were homogenized with a TissueLyser (Qiagen). Predeveloped, validated primer/probe sets were used (Applied Biosystems). Reactions were run in duplicate on an ABI Prism 7900HT with thermal cycling parameters specific for 1-step RT-PCR. The efficiency (slopes) of the target amplification and the efficiency of the reference endogenous control (GAPDH) amplification were $100 \%( \pm 10 \%)$. For sorted lung epithelial cells, the RNAqueous-Micro Total RNA Isolation kit (Thermo Fisher Scientific) was used with elution in a $20-\mu 1$ volume; 500 ng RNA was converted to cDNA.

Mouse exposures and harvests. Mice were intratracheally administered $S$. pneumoniae, serotype $3\left(5 \times 10^{4}\right.$ to $2 \times 10^{5} \mathrm{CFU}$, or $5 \times 10^{3} \mathrm{CFU}$ for survival studies) (ATCC). Lung was homogenized in PBS, and serial dilutions plated on blood agar for bacterial quantification. BALF was spun at $300 \mathrm{~g}$ for 6 minutes. Erythrocytes were lysed in hypotonic buffer on ice for 1 minute. Cells were then resuspended in PBS and counted. Cytospins were stained and differentials counted by light microscopy.

Cell sorting. Lungs were perfused, inflated, and digested with elastase (Worthington Biochemicals) for 45 minutes at $37^{\circ} \mathrm{C}$. Minced lung was then digested with DNase (Sigma-Aldrich) for 15 minutes. Cells were strained (70- $\mu \mathrm{m}$ filter), depleted of $\mathrm{CD} 45^{+}$cells using AutoMACS column (Miltenyi Biotec), and then blocked with anti-mouse CD16/CD32 (2.4G2) and normal mouse and rat serum (Jackson ImmunoResearch). Cells were incubated with one or more of the following antibodies/markers: CD34 (clone MEC14.7), CD45 (clone 104), EpCAM/CD326 (clone G8.8), CD31 (clone 390), CD103 (clone 2E7), 7-AAD (all Biolegend). Cells were then sorted (7-AAD-CD31-CD34-CD45-EpCAM ${ }^{+}$) on an ARIA-II flow cytometer (BD Biosciences) with purity greater than $92 \%-97 \%$.

Cytokine analysis. Cytokines were quantified by multiplex assay (Bio-Plex; Bio-Rad) or ELISA (eBioscience). BAFF (MBLYSO) and CXCL13 (MCX130) ELISAs (R\&D Systems) were used as directed. Lung homogenate was processed using the Bioplex Cell Lysis kit (Bio-Rad), and equivalent protein was used for each sample, as quantified by BCA.

$B C A$ protein assay. Manufacturer's instructions (Pierce) were followed, with colorimetric reading at OD562 on a Bio-Tek Synergy 2 microplate reader.

Immunoblotting. Saline-perfused lungs were homogenized in $0.1 \%$ Triton X-100 supplemented with protease inhibitors and then clarified by centrifugation. An equal protein mass of lung or BALF (first concentrated using a 3-kDa MW cutoff [Amicon Ultra-0.5 centrifugal filter unit with ultracel-3 membrane; MilliporeSigma]) was subjected to SDS-polyacrylamide gel electrophoresis and nitrocellulose membrane transfer as previously reported (51). For BALF IgA immunoblot, reducing agents were omitted from sample buffer. Membranes were probed with mouse anti- $\beta$-actin-peroxidase (1:25,000; SigmaAldrich, A3854), goat anti-IgA HRP (1:1,000; Abcam, ab97235), or goat anti-pIgR (1:1,000; R\&D Systems/Bio-techne, AF2800). Membranes were then washed and the latter target exposed (60 minutes) to 1:5,000 HRP-conjugated secondary antibody (GE Healthcare) in 5\% milk/buffer. After further washes, signal was detected with ECL Western Blot detection reagents (GE Healthcare), followed by film exposure (GE Healthcare).

Anti-PC and anti-T15 idiotype ELISAs. High-binding enzyme immunoassay/RIA plates (Costar) were coated with $2 \mu \mathrm{g} / \mathrm{ml}$ PC-BSA (Biosearch Tech) or $2 \mu \mathrm{g} / \mathrm{ml} \mathrm{AB1-2} \mathrm{(anti-Vk22} \mathrm{antibody)} \mathrm{in} \mathrm{PBS} \mathrm{and} \mathrm{incu-}$ bated overnight at $4^{\circ} \mathrm{C}$. Wells were washed and blocked in $1 \%$ BSA in PBS for 1 hour at room temperature. Purified monoclonal unlabeled antibodies provided by the Kearney laboratory (BH8 [anti-PC IgM] or S107 [anti-PC IgA]) were used as standards in some experiments where values are expressed as ng/ml. After 
sample and standard addition, plates were incubated overnight at $4^{\circ} \mathrm{C}$. The following day, the plates were washed and anti-mouse isotype antibody conjugated to alkaline phosphatase (Southern Biotechnology) was added at 1:1,000 dilution for 1 hour. Plates were then developed with BCIP-NBT (Sigma-Aldrich) and read at $405 \mathrm{~nm}$ on a Bio-Tek Synergy 2 microplate reader.

Statistics. Analysis was performed using GraphPad Prism software. Data are represented as the mean \pm SEM. Two-tailed Student's $t$ test was applied for comparisons of 2 groups and ANOVA for comparisons of more than 2 groups. Survival was evaluated by log-rank test. For all tests, $P$ less than 0.05 was considered significant.

Study approval. All experiments were performed in accordance with the Animal Welfare Act and the U.S. Public Health Service Policy on Humane Care and Use of Laboratory Animals after review by the National Institute of Environmental Health Sciences Animal Care and Use Committee.

\section{Author contributions}

$\mathrm{KA}, \mathrm{KG}, \mathrm{PR}$, and $\mathrm{HJ}$ designed, conducted, and analyzed experiments and contributed to the writing of the manuscript. NC, WC, JM, and LL contributed to tissue harvest, processing, and/or evaluation. KJ contributed to IHC, pathology evaluation, interpretation/report, and manuscript review. DC, MF, NC, PP, JK, and GT provided critical reagents, contributed to experimental design and interpretation, and contributed to the writing of the manuscript.

\section{Acknowledgments}

The authors thank Ligon Perrow for assistance with breeding; Tiwanda Masinde, Yvette Rebolloso, Geoffrey Hurbert, and David Olson (NIEHS IHC core); the NIEHS necropsy and histology core; Cristina Evans and David Goulding (colony support); Deborah King for blood cell count analysis; Ralph Wilson for clinical chemistry analysis; Carl Bortner and Maria Sifre for flow cytometry assistance; Jeff Tucker and Agnes Janoshazi from the Fluorescence Microscopy and Imaging Center for metamorphic analysis; Emily Mesev for assistance with immunoblotting; and David Kurtz and Jacqueline Locklear from the NIEHS QA Laboratory. We thank Marilyn Diaz and Chuancang Jiang for technical support with ANA and immunoglobulin ELISAs. We are indebted to the National Gnotobiotic Rodent Resource Center, UNC (5-P39DK034987, 5-P40-OD010995). This research was supported by the Intramural Research Program of the NIH, National Institute of Environmental Health Sciences (Z01 ES102005), and by NIH National Institute of Allergy and Infectious Diseases grants AI14782 and AI124072 (to JFK).

Address correspondence to: Michael B. Fessler, National Institute of Environmental Health Sciences, 111 T.W. Alexander Drive, P.O. Box 12233, MD D2-01, Research Triangle Park, North Carolina 27709, USA. Phone: 919.541.3701; Email: fesslerm@niehs.nih.gov.

1. Nocturne G, Mariette X. Advances in understanding the pathogenesis of primary Sjögren's syndrome. Nat Rev Rheumatol. 2013;9(9):544-556.

2. Delaleu N, Nguyen CQ, Peck AB, Jonsson R. Sjögren's syndrome: studying the disease in mice. Arthritis Res Ther. 2011;13(3):217.

3. Kreider M, Highland K. Pulmonary involvement in Sjögren syndrome. Semin Respir Crit Care Med. 2014;35(2):255-264.

4. Randall TD. Bronchus-associated lymphoid tissue (BALT) structure and function. Adv Immunol. 2010;107:187-241

5. Moyron-Quiroz JE, et al. Role of inducible bronchus associated lymphoid tissue (iBALT) in respiratory immunity. Nat Med. 2004;10(9):927-934.

6. Rangel-Moreno J, Hartson L, Navarro C, Gaxiola M, Selman M, Randall TD. Inducible bronchus-associated lymphoid tissue (iBALT) in patients with pulmonary complications of rheumatoid arthritis. J Clin Invest. 2006;116(12):3183-3194.

7. Howard J. The IRG proteins: a function in search of a mechanism. Immunobiology. 2008;213(3-4):367-375.

8. Hunn JP, Feng CG, Sher A, Howard JC. The immunity-related GTPases in mammals: a fast-evolving cell-autonomous resistance system against intracellular pathogens. Mamm Genome. 2011;22(1-2):43-54.

9. Feng CG, et al. Mice deficient in LRG-47 display increased susceptibility to mycobacterial infection associated with the induction of lymphopenia. J Immunol. 2004;172(2):1163-1168.

10. Hunn JP, Howard JC. The mouse resistance protein Irgm1 (LRG-47): a regulator or an effector of pathogen defense? PLoS Pathog. 2010;6(7):e1001008.

11. Traver MK, et al. Immunity-related GTPase M (IRGM) proteins influence the localization of guanylate-binding protein 2 (GBP2) by modulating macroautophagy. J Biol Chem. 2011;286(35):30471-30480.

12. Maric-Biresev J, Hunn JP, Krut O, Helms JB, Martens S, Howard JC. Loss of the interferon- $\gamma$-inducible regulatory immunityrelated GTPase (IRG), Irgm1, causes activation of effector IRG proteins on lysosomes, damaging lysosomal function and 
predicting the dramatic susceptibility of Irgm1-deficient mice to infection. BMC Biol. 2016;14:33.

13. Pilla-Moffett D, Barber MF, Taylor GA, Coers J. Interferon-inducible GTPases in host resistance, inflammation and disease. J Mol Biol. 2016;428(17):3495-3513.

14. Briles DE, Forman C, Hudak S, Claflin JL. Anti-phosphorylcholine antibodies of the T15 idiotype are optimally protective against Streptococcus pneumoniae. J Exp Med. 1982;156(4):1177-1185.

15. Feng CG, Zheng L, Lenardo MJ, Sher A. Interferon-inducible immunity-related GTPase Irgm1 regulates IFN gamma-dependent host defense, lymphocyte survival and autophagy. Autophagy. 2009;5(2):232-234.

16. Henry SC, Traver M, Daniell X, Indaram M, Oliver T, Taylor GA. Regulation of macrophage motility by Irgm1. J Leukoc Biol. 2010;87(2):333-343.

17. Shaw PX, et al. Natural antibodies with the T15 idiotype may act in atherosclerosis, apoptotic clearance, and protective immunity. J Clin Invest. 2000;105(12):1731-1740.

18. Feng CG, Weksberg DC, Taylor GA, Sher A, Goodell MA. The p47 GTPase Lrg-47 (Irgm1) links host defense and hematopoietic stem cell proliferation. Cell Stem Cell. 2008;2(1):83-89.

19. Baumgarth N. The double life of a B-1 cell: self-reactivity selects for protective effector functions. Nat Rev Immunol. 2011;11(1):34-46.

20. Lamagna C, Scapini P, van Ziffle JA, DeFranco AL, Lowell CA. Hyperactivated MyD88 signaling in dendritic cells, through specific deletion of Lyn kinase, causes severe autoimmunity and inflammation. Proc Natl Acad Sci USA. 2013;110(35):E3311-E3320.

21. Mariz HA, Sato EI, Barbosa SH, Rodrigues SH, Dellavance A, Andrade LE. Pattern on the antinuclear antibody-HEp-2 test is a critical parameter for discriminating antinuclear antibody-positive healthy individuals and patients with autoimmune rheumatic diseases. Arthritis Rheum. 2011;63(1):191-200.

22. Nordmark G, Alm GV, Rönnblom L. Mechanisms of disease: primary Sjögren's syndrome and the type I interferon system. Nat Clin Pract Rheumatol. 2006;2(5):262-269.

23. Kyriakidis NC, Kapsogeorgou EK, Gourzi VC, Konsta OD, Baltatzis GE, Tzioufas AG. Toll-like receptor 3 stimulation promotes Ro52/TRIM21 synthesis and nuclear redistribution in salivary gland epithelial cells, partially via type I interferon pathway. Clin Exp Immunol. 2014;178(3):548-560.

24. Salvi S, Holgate ST. Could the airway epithelium play an important role in mucosal immunoglobulin A production? Clin Exp Allergy. 1999;29(12):1597-1605.

25. Shimada S, et al. Generation of polymeric immunoglobulin receptor-deficient mouse with marked reduction of secretory IgA. J Immunol. 1999;163(10):5367-5373.

26. Masmoudi H, Mota-Santos T, Huetz F, Coutinho A, Cazenave PA. All T15 Id-positive antibodies (but not the majority of VHT15 ${ }^{+}$antibodies) are produced by peritoneal CD5+ B lymphocytes. Int Immunol. 1990;2(6):515-520.

27. Binder CJ, Silverman GJ. Natural antibodies and the autoimmunity of atherosclerosis. Springer Semin Immunopathol. 2005;26(4):385-404.

28. Xu Z, Morel L. Contribution of B-1a cells to systemic lupus erythematosus in the NZM2410 mouse model. Ann N Y Acad Sci. 2015;1362:215-223.

29. Baldan A, et al. ABCG1 is required for pulmonary B-1 B cell and natural antibody homeostasis. J Immunol. 2014;193(11):5637-5648.

30. Benedict CL, Gilfillan S, Thai TH, Kearney JF. Terminal deoxynucleotidyl transferase and repertoire development. Immunol Rev. 2000;175:150-157.

31. Afzelius P, Fallentin EM, Larsen S, Møller S, Schiødt M. Pancreatic function and morphology in Sjögren's syndrome. Scand J Gastroenterol. 2010;45(6):752-758.

32. Ito $\mathrm{T}$, et al. Defective B1 cell homing to the peritoneal cavity and preferential recruitment of B1 cells in the target organs in a murine model for systemic lupus erythematosus. J Immunol. 2004;172(6):3628-3634.

33. Ansel KM, Harris RB, Cyster JG. CXCL13 is required for B1 cell homing, natural antibody production, and body cavity immunity. Immunity. 2002;16(1):67-76.

34. Kroese FG, Butcher EC, Stall AM, Lalor PA, Adams S, Herzenberg LA. Many of the IgA producing plasma cells in murine gut are derived from self-replenishing precursors in the peritoneal cavity. Int Immunol. 1989;1(1):75-84.

35. Kramer JM, Klimatcheva E, Rothstein TL. CXCL13 is elevated in Sjögren's syndrome in mice and humans and is implicated in disease pathogenesis. J Leukoc Biol. 2013;94(5):1079-1089.

36. Pourmand N, et al. Ro/SSA and La/SSB specific IgA autoantibodies in serum of patients with Sjögren's syndrome and systemic lupus erythematosus. Ann Rheum Dis. 1999;58(10):623-629.

37. Xu H, et al. Genetic deficiency of Irgm1 (LRG-47) suppresses induction of experimental autoimmune encephalomyelitis by promoting apoptosis of activated CD4 ${ }^{+}$T cells. FASEB J. 2010;24(5):1583-1592.

38. Bafica A, et al. The IFN-inducible GTPase LRG47 (Irgm1) negatively regulates TLR4-triggered proinflammatory cytokine production and prevents endotoxemia. J Immunol. 2007;179(8):5514-5522.

39. Gilliet M, Cao W, Liu YJ. Plasmacytoid dendritic cells: sensing nucleic acids in viral infection and autoimmune diseases. Nat Rev Immunol. 2008;8(8):594-606.

40. He B, et al. The transmembrane activator TACI triggers immunoglobulin class switching by activating B cells through the adaptor MyD88. Nat Immunol. 2010;11(9):836-845.

41. Chu VT, Enghard P, Riemekasten G, Berek C. In vitro and in vivo activation induces BAFF and APRIL expression in B cells. J Immunol. 2007;179(9):5947-5957.

42. Groom JR, et al. BAFF and MyD88 signals promote a lupuslike disease independent of T cells. J Exp Med. 2007;204(8):1959-1971.

43. Sun L, et al. Type I interferons link viral infection to enhanced epithelial turnover and repair. Cell Host Microbe. 2015;17(1):85-97.

44. Rivas MN, et al. MyD88 is critically involved in immune tolerance breakdown at environmental interfaces of Foxp3-deficient mice. J Clin Invest. 2012;122(5):1933-1947.

45. Martinez J, et al. Noncanonical autophagy inhibits the autoinflammatory, lupus-like response to dying cells. Nature. 2016;533(7601):115-119.

46. Weindel CG, Richey LJ, Bolland S, Mehta AJ, Kearney JF, Huber BT. B cell autophagy mediates TLR7-dependent autoimmunity 
and inflammation. Autophagy. 2015;11(7):1010-1024.

47. Arnold J, Murera D, Arbogast F, Fauny JD, Muller S, Gros F. Autophagy is dispensable for B-cell development but essential for humoral autoimmune responses. Cell Death Differ. 2016;23(5):853-864

48. Clarke AJ, et al. Autophagy is activated in systemic lupus erythematosus and required for plasmablast development. Ann Rheum Dis. 2015;74(5):912-920.

49. Gros F, et al. Macroautophagy is deregulated in murine and human lupus T lymphocytes. Autophagy. 2012;8(7):1113-1123.

50. Fessler MB, Summer RS. Surfactant lipids at the host-environment interface. Metabolic sensors, suppressors, and effectors of inflammatory lung disease. Am J Respir Cell Mol Biol. 2016;54(5):624-635.

51. Draper DW, et al. ATP binding cassette transporter G1 deletion induces IL-17-dependent dysregulation of pulmonary adaptive immunity. J Immunol. 2012;188(11):5327-5336 